\title{
DIFFUSION INDUCED BY GRAIN BOUNDARIES: A SHE MODEL*
}

\author{
NAOUFEL BEN ABDALLAH ${ }^{\dagger}$ AND HÉDIA CHAKER $\ddagger$
}

\begin{abstract}
Classical motion of electrons in a two dimensional superlattice is considered. The lattice unit cell is a square with a small side. When electrons hit a cell side, they are reflected with a probability $\mathcal{R}$ and transmitted with probability $\mathcal{T}$. A diffusion approximation of the model is performed and leads to a diffusion equation in position-energy variables ( SHE model). The diffusion constant can be expressed explicitly in terms of reflection-transmission coefficients. The mathematical problem is a two dimensional version of a previous work in the one dimensional case [ $\mathrm{N}$. Ben Abdallah, P. Degond, A. Mellet, F. Poupaud, Electron transport in semiconductor superlattices, Quarterly Appl. Math. 2003, 61 (1) 161-192], and appears in the modeling of gas sensors.
\end{abstract}

\section{Introduction}

Polycrystalline semiconductors, in thin layers, are used in many technological applications such as gas detectors [20,21,24]. The polycrystal is a collection of microscopic crystal grains separated from each other by very thin regions called grain boundaries. At the grain boundary, charges can be trapped thus creating and/or modulating a localized potential barrier. This in turn modifies the probability that an electron hitting the grain boundary is transmitted to a neighboring grain or reflected back. The reflection-transmission phenomenon is responsible for the surface conductivity of the gas sensor, and is used to measure the concentration of the gas [20]. The principle of operation is as follows: as the gas molecules are deposited on the sensor surface, they are ionized and adsorbed. They are more likely to be trapped at the grain boundaries. The value of the trapped charge is directly related to the gas concentration; this relationship depends on the adsorption mechanism [5, 27]. Of course, the higher the gas concentration is the bigger the trapped charge. Its value, together with the doping concentration and the macroscopic electron density, determine the electrostatic structure at the grain boundary. This in turn determines the reflectiontransmission coefficients across the grain boundary from which the conductivity of the gas sensor can be deduced. To summarize, the value of the gas concentration determines the conductivity of the sensor. By measuring the current flowing through the sensor, its conductivity is measured which allows us to deduce the gas concentration. The aim of this paper is to show how the conductivity can be deduced from the reflection-transmission coefficients of the grain boundaries. This is done by deriving a diffusion model whose diffusion coefficients are directly expressed in terms of the reflection-transmision coefficients. The grains are assumed to be arranged in a periodic bidimensional lattice, and the scattering coefficients at the grain boundaries are assumed to be known. We do not consider here the way to compute these coefficients from the electrical structure of the grain boundary. The scaled length of a simple grain is denoted by $\alpha$ and is assumed to be very small. Electrons are submitted to a macroscopic electrostatic potential and flow according to Newton's law in the grain. When they hit the grain boundary, they have a probability $\mathcal{R}$ to be reflected back following Descartes law and a probability $\mathcal{T}$ to be tranmitted to the neighboring grain

\footnotetext{
${ }^{*}$ Received: July 8, 2003; accepted (in revised version): February 10, 2004. Communicated by Shi Jin.

† Mathématiques pour l'Industrie et la Physique Unité Mixte de Recherche 5640 (CNRS), Université Paul Sabatier 118, route de Narbonne, 31062 Toulouse Cedex, France, (naoufel@mip.ups-tlse.fr).

${ }^{\ddagger}$ Laboratoire de Modélisation mathématique et Numérique dans les Sciences de l’INgénieur, Ecole Nationale d'Ingénieurs de Tunis BP 37, Campus Universitaire, Le Belvédère, 1002, Tunis, Tunisie, (hedia.chaker@enit.rnu.tn).
} 
without changing their velocity. Since the grains are very small, electrons undergo a large number of collisions with grain boundaries which drive them towards a diffusive motion, and because the interaction is elastic (the kinetic energy is conserved during the collision with the grain boundary), diffusion occurs not only in position but also in energy variables. The diffusion model that is obtained is the so-called Spherical Harmonics Expansion (SHE) model which is now a standard transport model in semiconductors $[17,18,19,25]$. It has been derived for relaxation collision operators in bulk semiconductors [16] and then generalized to general collision operators in [2]. In $[6,7,8,9,11,12,13]$, The SHE model is derived in many other areas of charge particle transport such as plasmas or gas discharges. In these references, volume and surface collisions are considered. In [4], a one dimensional SHE model is derived for electron transport in semiconductor superlattices as a diffusion limit of the Vlasov equation with transmission-reflection interface conditions. The SHE model has also been obtained from a discrete transfer matrix model [14, 15].

The present work is a two dimensional generalization of [4]. The outline of the paper is the following. In Section 2, the setting of the problem, the hypotheses as well as the main results are given. The SHE model being the limit as the length size $\alpha$ of the grain sides tends to zero, Section 3 is devoted to the study of the problem for a positive $\alpha$. In Section 4, uniform estimates in $\alpha$ are obtained and the limit $\alpha \rightarrow 0$ is performed in Section 5. Finally, an extension of the result is developed in Section 6 . The mathematical arguments involved in the proofs of Sections 3,4 and 5 are similar to the one dimensional case [4]. We shall only develop in detail the specific issues raised by the bidimensional setting.

\section{Setting of the problem and main results}

The geometry of the problem is bidimensional. The position coordinates are denoted by $x=\left(x_{1}, x_{2}\right) \in \mathbb{R}^{2}$. The grains are assumed to be squares of side $\alpha$ arranged periodically. Each grain occupies a cell $I_{n, m}=[n \alpha,(n+1) \alpha] \times[m \alpha,(m+1) \alpha]$. The grain boundaries are the interfaces between two adjacent grains. The vertical interfaces $V_{n, m}$ are located at $\left(x_{1}=n \alpha ; x_{2} \in[m \alpha,(m+1) \alpha]\right)$ while the horizontal onces $H_{n, m}$ are located at $\left(x_{1} \in[n \alpha,(n+1) \alpha] ; x_{2}=m \alpha\right)$ with $n \in \mathbb{Z}$ and $m \in \mathbb{Z}$ (see Fig 2.1). We shall use the notation $V_{n}=\cup_{m \in \mathbb{Z}} V_{n, m}=n \alpha \times \mathbb{R}$ and $H_{m}=$ $\cup_{n \in \mathbb{Z}} H_{n, m}=\mathbb{R} \times m \alpha$

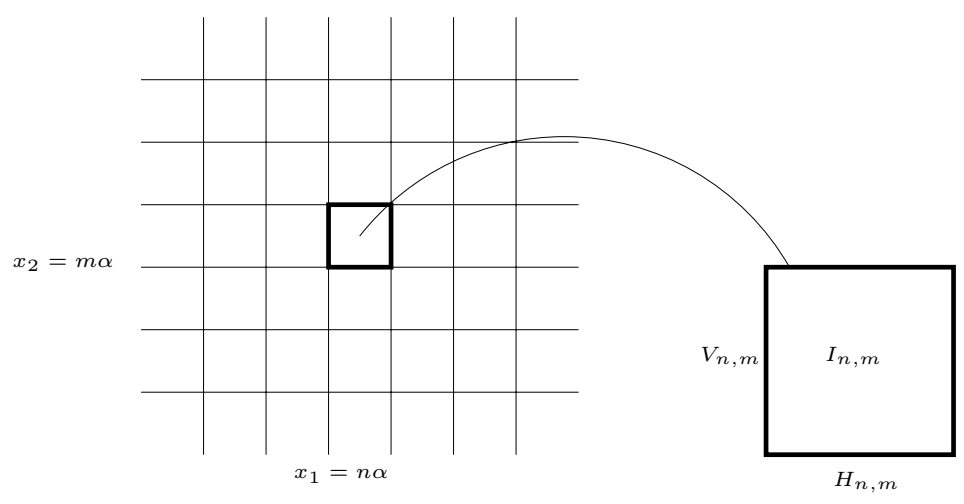

FIG. 2.1. unit cells, vertical and horizontal interfaces

The electron distribution function is denoted by $f(t, x, v)$; the position variable 
$x=\left(x_{1}, x_{2}\right)$ belongs to $\Omega=\mathbb{R}^{2} / L_{\alpha}$ where $L_{\alpha}=\alpha \mathbb{Z} e_{1} \times \mathbb{R} e_{2} \cup \mathbb{R} e_{1} \times \alpha \mathbb{Z} e_{2}$, the velocity $v=\left(v_{1}, v_{2}\right)$ is in $\mathbb{R}^{2}$, while the time variable $t$ belongs to $[0, \infty)$. In the grains, the distribution function $f$ is a solution of the scaled collisionless Vlasov equation

$$
\alpha \partial_{t} f^{\alpha}+v \cdot \nabla_{x} f^{\alpha}+\nabla_{x} V \cdot \nabla_{v} f^{\alpha}=0, \quad x \in \Omega^{\alpha}, v \in \mathbb{R}^{2}, t \geq 0,
$$

where $V=V(x)$ is the electrostatic potential assumed to be a known, stationary, regular function varying over the macroscopic scale. For any given function $\varphi(x, v)$, the left, right, upper and lower traces of $\varphi$ on the grain boundaries are defined by

$$
\begin{gathered}
\varphi_{V_{n}}^{\mp}\left(x_{2}, v\right)=\gamma_{V_{n}}^{\mp}(\varphi)\left(x_{2}, v\right)=\lim _{x_{1} \rightarrow n \alpha \mp} \varphi(x, v), \\
\varphi_{H_{m}}^{\mp}\left(x_{1}, v\right)=\gamma_{H_{m}}^{\mp}(\varphi)\left(x_{1}, v\right)=\lim _{x_{2} \rightarrow m \alpha \mp} \varphi(x, v),
\end{gathered}
$$

while the outgoing and incoming traces are given by

$$
\begin{gathered}
\gamma_{V_{n}}^{\text {out }}(\varphi)\left(x_{2}, v\right)= \begin{cases}\varphi_{V_{n}}^{-}\left(x_{2}, v\right), & \text { if } v_{1}>0, \\
\varphi_{V_{n}}^{+}\left(x_{2}, v\right), & \text { if } v_{1}<0,\end{cases} \\
\gamma_{V_{n}}^{\text {inc }}(\varphi)\left(x_{2}, v\right)= \begin{cases}\varphi_{V_{n}}^{-}\left(x_{2}, v\right), & \text { if } v_{1}<0, \\
\varphi_{V_{n}}^{+}\left(x_{2}, v\right), & \text { if } v_{1}>0,\end{cases} \\
\gamma_{H_{m}}^{\text {out }}(\varphi)\left(x_{1}, v\right)= \begin{cases}\varphi_{H_{m}}^{-}\left(x_{1}, v\right), & \text { if } v_{2}>0, \\
\varphi_{H_{m}}^{+}\left(x_{1}, v\right), & \text { if } v_{2}<0,\end{cases} \\
\gamma_{H_{m}}^{\text {inc }}(\varphi)\left(x_{1}, v\right)= \begin{cases}\varphi_{H_{m}}^{-}\left(x_{1}, v\right), & \text { if } v_{2}<0, \\
\varphi_{H_{m}}^{+}\left(x_{1}, v\right), & \text { if } v_{2}>0 .\end{cases}
\end{gathered}
$$

The grain boundary is assumed to behave as an electrostatic barrier or well which has a fast transversal dependence and a slow parallel one. Therefore, an electron hitting the grain boundary has a probability $\mathcal{T}$ to be transmitted to the neighboring cell and a probability $\mathcal{R}$ to be reflected back following Descartes law (see Figure 2.2). The reflection and transmission coefficients $\mathcal{R}$ and $\mathcal{T}$ depend on the microstructure of the grain boundary and are functions of the electron energy and position. They can be computed by solving the Schrödinger equation [3]. The boundary condition for the distribution function $f^{\alpha}$ is then written in terms of these coefficients

$$
\begin{aligned}
\gamma_{V_{n}}^{\text {inc }} f^{\alpha} & =\mathcal{B}^{V}\left(n \alpha, x_{2}\right) \gamma_{V_{n}}^{\text {out }} f^{\alpha}, \\
\gamma_{H_{m}}^{\text {inc }} f^{\alpha} & =\mathcal{B}^{H}\left(x_{1}, m \alpha\right) \gamma_{H_{m}}^{\text {out }} f^{\alpha},
\end{aligned}
$$

where the operators $\mathcal{B}^{V}$ and $\mathcal{B}^{H}$ are defined by

$$
\begin{aligned}
& \left(\mathcal{B}^{V}(x) \varphi\right)(v)=\mathcal{R}^{V}(x, v) \varphi\left(x,-v_{1}, v_{2}\right)+\mathcal{T}^{V}(x, v) \varphi(x, v), \\
& \left(\mathcal{B}^{H}(x) \varphi\right)(v)=\mathcal{R}^{H}(x, v) \varphi\left(x, v_{1},-v_{2}\right)+\mathcal{T}^{H}(x, v) \varphi(x, v) .
\end{aligned}
$$



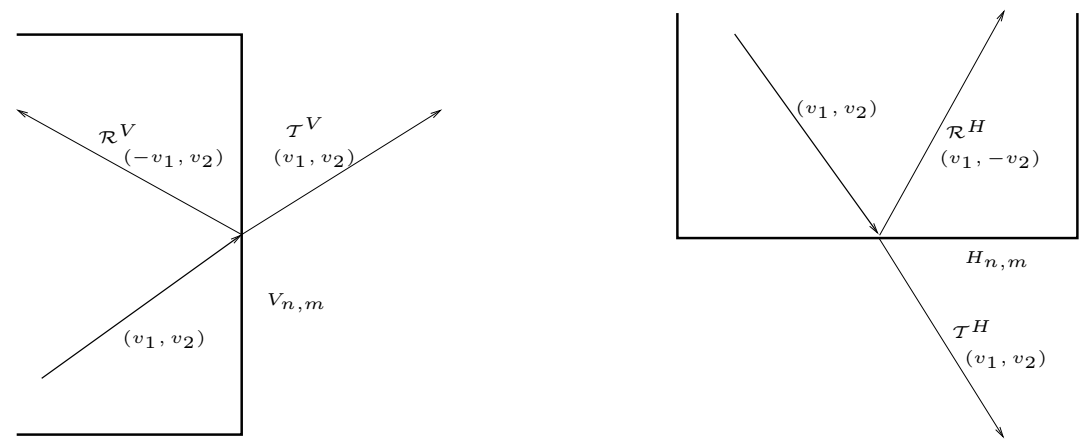

FIG. 2.2. Reflection-transmission picture at a grain boundary

In the sequel, we shall sometimes use the short notation

$$
\mathcal{B}(x)=\mathcal{B}^{V}(x) \text { for } x \in \cup_{n \in \mathbb{Z}} V_{n} ; \quad \mathcal{B}(x)=\mathcal{B}^{H}(x) \text { for } x \in \cup_{m \in \mathbb{Z}} H_{m}
$$

REMARK 2.1. $\mathcal{B}^{V}(x)$ only acts on the $v_{1}$ variable, the variable $v_{2}$ being only a parameter. Analogously, $v_{1}$ is only a parameter for $\mathcal{B}^{H}(x)$ which acts on the $v_{2}$ variable only.

The aim of the paper is the analysis of the limit $\alpha \rightarrow 0$ of the kinetic model (2.1), (2.2), (2.3), with the initial data

$$
f^{\alpha}(t=0, x, v)=f_{I}^{\alpha}(x, v), \quad x \in \Omega^{\alpha}, v \in \mathbb{R}^{2} .
$$

To avoid the treatment of initial layers, we shall assume that the initial datum is well prepared. Namely we assume

Hypothesis 2.1. There exists a smooth function $F_{I}\left(x, \varepsilon_{1}, \varepsilon_{2}\right)$ defined on $\mathbb{R}^{2} \times \mathbb{R}_{+}^{2}$, satisfying:

$$
F_{I}\left(x, \frac{v_{1}^{2}}{2}, \frac{v_{2}^{2}}{2}\right) \in L^{2}\left(\mathbb{R}^{2} \times \mathbb{R}_{+}^{2}\right), \quad\left(v \cdot \nabla_{x}+\nabla_{x} V \cdot \nabla_{v}\right) F_{I}\left(x, \frac{v_{1}^{2}}{2}, \frac{v_{2}^{2}}{2}\right) \in L^{2}\left(\mathbb{R}^{2} \times \mathbb{R}_{+}^{2}\right),
$$

such that

$$
f_{I}^{\alpha}(x, v)=F_{I}\left(x, \frac{v_{1}^{2}}{2}, \frac{v_{2}^{2}}{2}\right), \quad x \in \Omega^{\alpha}, v \in \mathbb{R}^{2} .
$$

The electrostatic potential is assumed to satisfy the following hypothesis

Hypothesis 2.2. The electric field $\nabla_{x} V$ belongs to the Sobolev space $W^{1, \infty}\left(\mathbb{R}^{2}\right)^{2}$.

HYPOTHESIS 2.3. The reflection and transmission coefficients are even functions of $v_{1}$ and $v_{2}$ and $C^{1}$ functions of $\left(x, \varepsilon_{1}, \varepsilon_{2}\right)$, where $\varepsilon_{i}=\frac{v_{i}^{2}}{2}$. They satisfy the positivity property : $0<\mathcal{T}^{H, V}\left(x, \varepsilon_{1}, \varepsilon_{2}\right)<1, \quad \forall\left(x, \varepsilon_{1}, \varepsilon_{2}\right) \in \mathbb{R}^{2} \times \mathbb{R}_{+}^{2}$ as well as the identity $\mathcal{T}^{H, V}+\mathcal{R}^{H, V} \equiv 1$. We therefore write, $\mathcal{R}^{H, V}=\mathcal{R}^{H, V}\left(x, \varepsilon_{1}, \varepsilon_{2}\right)$ (respectively $\mathcal{T}^{H, V}=$ $\mathcal{T}^{H, V}\left(x, \varepsilon_{1}, \varepsilon_{2}\right)$ instead of $\mathcal{R}^{H, V}\left(x, v_{1}, v_{2}\right)$ ( respectively $\mathcal{T}^{H, V}\left(x, v_{1}, v_{2}\right)$ ).

The main result of this paper is the following

Theorem 2.1. i) Under Hypotheses 2.1, 2.2, 2.3, the problem (2.1),(2.2), (2.3) has a solution $f^{\alpha}$ (in a sense that will be specified further, see Proposition 4.2). 
ii) When $\alpha$ tends to zero, $f^{\alpha}$ converges (up to the extraction of a subsequence) in the weak star topology of $L^{\infty}\left([0, T], L^{2}\left(\mathbb{R}^{2} \times \mathbb{R}^{2}\right)\right)$ for any $T>0$. There exists a function $F\left(t, x, \varepsilon_{1}, \varepsilon_{2}\right)$ such that the limit $f^{0}(t, x, v)=F\left(t, x, \frac{v_{1}^{2}}{2}, \frac{v_{2}^{2}}{2}\right)$. It is the solution of the problem (SHE model) posed on the domain $\left(x, \varepsilon_{1}, \varepsilon_{2}\right) \in \mathbb{R}^{2} \times \mathbb{R}_{+}^{2}$ :

$$
\begin{aligned}
& \frac{2}{\sqrt{\varepsilon_{1} \varepsilon_{2}}} \partial_{t} F+\left(\partial_{x_{1}}+\partial_{x_{1}} V \partial_{\varepsilon_{1}}\right) J_{1}+\left(\partial_{x_{2}}+\partial_{x_{2}} V \partial_{\varepsilon_{2}}\right) J_{2}=0, \\
& J_{1}=-D_{11}\left(\partial_{x_{1}}+\partial_{x_{1}} V \partial_{\varepsilon_{1}}\right) F, \quad J_{2}=-D_{22}\left(\partial_{x_{2}}+\partial_{x_{2}} V \partial_{\varepsilon_{2}}\right) F, \\
& \partial_{x_{1}} V J_{1}\left(t, x, 0, \varepsilon_{2}\right)=0, \\
& F\left(0, x, \varepsilon_{1}, \varepsilon_{2}\right)=F_{I}\left(x, \varepsilon_{1}, \varepsilon_{2}\right) .
\end{aligned}
$$

The diffusion coefficients $D_{11}, D_{22}$ are given by

$$
D_{11}=\frac{2}{\sqrt{2 \varepsilon_{2}}} \frac{\mathcal{T}^{V}}{\left(I-\mathcal{T}^{V}\right)}, \quad D_{22}=\frac{2}{\sqrt{2 \varepsilon_{1}}} \frac{\mathcal{T}^{H}}{\left(I-\mathcal{T}^{H}\right)} .
$$

\section{Existence of the solution}

3.1. Preliminaries. In this section, we introduce some notation and present existence results for the perturbation problem. The methodology is identical to the superlattice case developed in [4]. Therefore, many proofs are skipped. Following the notation of [4], we denote by $L_{\alpha}=\alpha \mathbb{Z} e_{1} \times \mathbb{R} e_{2} \cup \mathbb{R} e_{1} \times \alpha \mathbb{Z} e_{2}, \Omega^{\alpha}=\mathbb{R}^{2} / L_{\alpha}$, $\mathcal{O}^{\alpha}=\Omega^{\alpha} \times \mathbb{R}^{2}$ and $\Gamma^{\alpha}=L_{\alpha} \times \mathbb{R}^{2}$. $\mathcal{O}^{\alpha}$ is equipped with the usual $L^{2}$ norm and inner product. Note that $L^{2}\left(\mathcal{O}^{\alpha}\right)=L^{2}\left(\mathbb{R}^{2} \times \mathbb{R}^{2}\right)$ since $L_{\alpha} \times \mathbb{R}^{2}$ is a zero measure set. Then

$$
|u|_{L^{2}\left(\mathcal{O}^{\alpha}\right)}^{2}=\int_{\mathbb{R}^{2} \times \mathbb{R}^{2}}|u(x, v)|^{2} d x d v .
$$

We denote by $\left.L_{\delta, i}^{2}\left(\mathbb{R}^{2}\right)\right), i=1,2$, the weighted $L^{2}$ space, equipped with norm

$$
|u|_{L_{\delta, i}^{2}\left(\mathbb{R}^{2}\right)}^{2}=\int_{\mathbb{R}^{2}}|u(v)|^{2} \omega_{i}^{\delta}(v) d v,
$$

with $\omega_{1}^{\delta}(v)=v_{1} s^{\delta}\left(v_{1}\right) I^{\delta}\left(v_{2}\right)$ and $\omega_{2}^{\delta}(v)=v_{2} s^{\delta}\left(v_{2}\right) I^{\delta}\left(v_{1}\right)$, where $s^{\delta}(y)$ and $I^{\delta}(y)$ are the continuous piecewise linear functions defined by

$$
\begin{gathered}
s^{\delta}(y)= \begin{cases}\frac{y}{\delta}, & \text { if } 0 \leq|y| \leq \delta, \\
\operatorname{sgn}(y), & \text { if } \delta \leq|y| \leq \frac{1}{\delta}-\delta, \\
\frac{\operatorname{sgn}(y)}{\delta}\left(\frac{1}{\delta}-|y|\right), & \text { if } \frac{1}{\delta}-\delta \leq|y|, \leq \frac{1}{\delta}, \\
0 & \text { if } \frac{1}{\delta} \leq|y|,\end{cases} \\
I^{\delta}(y)= \begin{cases}1, & \text { if } 0 \leq|y| \leq \frac{1}{\delta}-\delta, \\
\frac{1}{\delta}\left(\frac{1}{\delta}-|y|\right), & \text { if } \frac{1}{\delta}-\delta \leq|y| \leq \frac{1}{\delta}, \\
0, & \text { if } \frac{1}{\delta} \leq|y| .\end{cases}
\end{gathered}
$$


where $s g n$ is the sign function. The space $L_{0, i}^{2}\left(\mathbb{R}^{2}\right)(i=1,2)$ is nothing but the weighted $L^{2}$ space associated with the weight $\left|v_{i}\right|$, which will be denoted by $L_{i}^{2}\left(\mathbb{R}^{2}\right)$. We denote by $L^{2}\left(\mathbb{R}_{x_{1}}, L_{\delta, 2}^{2}\left(\mathbb{R}^{2}\right)\right)$ the weighted $L^{2}$ space, equipped with norm

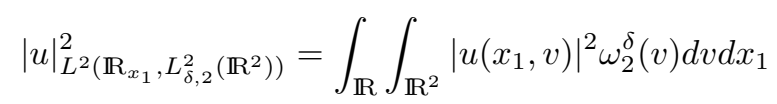

and $L^{2}\left(\mathbb{R}_{x_{2}}, L_{\delta, 1}^{2}\left(\mathbb{R}^{2}\right)\right)$ the weighted $L^{2}$ space, equipped with norm

$$
|u|_{L^{2}\left(\mathbb{R}_{x_{2}}, L_{\delta, 1}^{2}\left(\mathbb{R}^{2}\right)\right)}^{2}=\int_{\mathbb{R}} \int_{\mathbb{R}^{2}}\left|u\left(x_{2}, v\right)\right|^{2} \omega_{1}^{\delta}(v) d v d x_{2} .
$$

We denote by $L_{\delta}^{2}\left(\Gamma^{\alpha}\right)$ the set of functions $u=\left(u_{n \alpha}\left(x_{2}, v\right), u_{m \alpha}\left(x_{1}, v\right)\right)_{n \in \mathbb{Z}, m \in \mathbb{Z}}$ such that $u_{n \alpha}\left(x_{2}, v\right) \in L^{2}\left(\mathbb{R}_{x_{2}}, L_{\delta, 1}^{2}\left(\mathbb{R}^{2}\right)\right)$ for all $n \in \mathbb{Z}$ and $u_{m \alpha}\left(x_{1}, v\right) \in L^{2}\left(\mathbb{R}_{x_{1}}, L_{\delta, 2}^{2}\left(\mathbb{R}^{2}\right)\right)$ for all $m \in \mathbb{Z}$. This set is naturally equipped with the norm

$$
|u|_{L_{\delta}^{2}\left(\Gamma^{\alpha}\right)}=\left(\sum_{n \in \mathbb{Z}} \alpha\left|u_{n \alpha}\right|_{L^{2}\left(\mathbb{R}_{x_{2}}, L_{\delta, 1}^{2}\left(\mathbb{R}^{2}\right)\right)}^{2}+\sum_{m \in \mathbb{Z}} \alpha\left|u_{m \alpha}\right|_{L^{2}\left(\mathbb{R}_{x_{1}}, L_{\delta, 2}^{2}\left(\mathbb{R}^{2}\right)\right)}^{2}\right)^{\frac{1}{2}} .
$$

Proposition 3.1. i) For all $x \in \mathbb{R}^{2}$ and all $v_{2} \in \mathbb{R}$, we consider $\mathcal{B}^{V}$ as an operator on $L_{1}^{2}(\mathbb{R})$. It is self-adjoint and the null-space $N\left(I-\mathcal{B}^{V}\right)$ is the set of even functions with respect to $v_{1}$ while $\operatorname{Im}\left(I-\mathcal{B}^{V}\right)$ is the set of odd functions with respect to $v_{1}$.

ii) The same result holds for $\mathcal{B}^{H}$ considered as an operator on $L_{2}^{2}(\mathbb{R})$ by exchanging the roles of $v_{1}$ and $v_{2}$.

Proof: The result is immediate since $\left(I-\mathcal{B}^{V}\right) \phi=\mathcal{R}^{V}\left(x, \varepsilon_{1}, \varepsilon_{2}\right)\left(\phi\left(v_{1}, v_{2}\right)-\right.$ $\left.\phi\left(-v_{1}, v_{2}\right)\right)$.

Let us now denote by $Q_{V}$ ( respectively $\left.Q_{H}\right)$ the orthogonal projector of $L_{1}^{2}\left(\mathbb{R}^{2}\right)$ (respectively $L_{2}^{2}\left(\mathbb{R}^{2}\right)$ ) onto the the space of even functions with respect to $v_{1}$ (respectively $v_{2}$ ) and introduce $P_{V}=I-Q_{V}$ (respectively $P_{H}=I-Q_{H}$ ) where $I$ is the identity on $L_{1}^{2}\left(\mathbb{R}^{2}\right)$ (respectively $L_{2}^{2}\left(\mathbb{R}^{2}\right)$ ). For $x \in L_{\alpha}$, we define $Q(x)$ and $P(x)$ by

$$
Q(x)=\left\{\begin{array}{ll}
Q_{V}, & \text { on } \cup_{n \in \mathbb{Z}} V_{n}, \\
Q_{H}, & \text { on } \cup_{m \in \mathbb{Z}} H_{m},
\end{array} \quad P(x)=\left\{\begin{array}{ll}
P_{V}, & \text { on } \cup_{n \in \mathbb{Z}} V_{n}, \\
P_{H}, & \text { on } \cup_{m \in \mathbb{Z}} H_{m} .
\end{array} \quad ;(P=I-Q) .\right.\right.
$$

It is readily seen that

Lemma 3.1. The operators $Q, P$, and $B$ satisfy the following identities

$$
P \mathcal{B}=\mathcal{B} P, \quad Q \mathcal{B}=\mathcal{B} Q=Q
$$

Moreover, there exists $K_{1}\left(x, v_{2}\right)<1$ defined for $x \in \cup_{n \in \mathbb{Z}} V_{n}$ and $v_{2} \in \mathbb{R}$ ( respectively $K_{2}\left(x, v_{1}\right)<1$ defined for $x \in \cup_{m \in \mathbb{Z}} H_{m}$ and $\left.v_{1} \in \mathbb{R}\right)$ such that

$$
\left\|\mathcal{B}^{V}\left(x, v_{2}\right) P_{V}(x)\right\|_{\mathcal{L}\left(L_{1}^{2}(\mathbb{R})\right)} \leq K_{1}\left(x, v_{2}\right)<1,\left\|\mathcal{B}^{H}\left(x, v_{1}\right) P_{H}(x)\right\|_{\mathcal{L}\left(L_{2}^{2}(\mathbb{R})\right)} \leq K_{2}\left(x, v_{1}\right)<1 .
$$

Finally, we have

$$
\|\tilde{\mathcal{B}}\|_{\mathcal{L}\left(L_{1}^{2}(\mathbb{R}) \times L_{2}^{2}(\mathbb{R})\right)} \leq 1
$$


HyPOTHESIS 3.1. Let

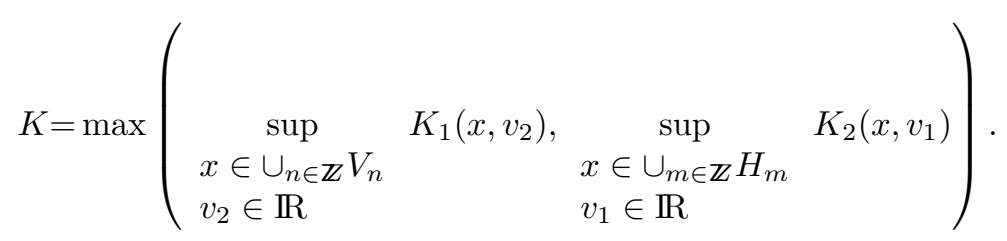

We assume that $K<1$.

Following the notations of [4], the transport operator is defined by

$$
\mathcal{A}^{\alpha} u=v \cdot \nabla_{x} u+\nabla_{x} V \cdot \nabla_{v} u
$$

on the domain $H^{\alpha}(\mathcal{A}, \mathcal{B})=\left\{u \in H^{\alpha}(\mathcal{A}), \gamma_{\alpha}^{\text {out }}(u) \in L^{2}\left(\Gamma^{\alpha}\right), \gamma_{\alpha}^{\text {inc }}(u)=\mathcal{B} \gamma_{\alpha}^{\text {out }}(u)\right\}$, where the space $H^{\alpha}(\mathcal{A})$ is defined by $H^{\alpha}(\mathcal{A})=\left\{u \in L^{2}\left(\mathcal{O}^{\alpha}\right), \mathcal{A}^{\alpha} u \in L^{2}\left(\mathcal{O}^{\alpha}\right)\right\}$. The spaces $H^{\alpha}(\mathcal{A})$ and $H^{\alpha}(\mathcal{A}, \mathcal{B})$ are equipped with the graph norm

$$
|u|_{H^{\alpha}(\mathcal{A})}^{2}=|u|_{L^{2}\left(\mathcal{O}^{\alpha}\right)}^{2}+\left|\mathcal{A}^{\alpha} u\right|_{L^{2}\left(\mathcal{O}^{\alpha}\right)}^{2} .
$$

We shall denote by $\mathcal{A}$ the bare differential operator, when no indication of the domain is needed. Defining

$$
H_{0}^{\alpha}(\mathcal{A})=\left\{u \in L^{2}\left(\mathcal{O}^{\alpha}\right), \mathcal{A}^{\alpha} u \in L^{2}\left(\mathcal{O}^{\alpha}\right), \gamma_{\alpha}^{\text {out }}(u) \in L^{2}\left(\Gamma^{\alpha}\right)\right\}
$$

We have the following Green's formula [1].

Lemma 3.2. (Green's formula) For $v, w$ in $H_{0}^{\alpha}(\mathcal{A})$, we have

$$
(\mathcal{A} u, v)_{L^{2}\left(\mathcal{O}^{\alpha}\right)}+(u, \mathcal{A} v)_{L^{2}\left(\mathcal{O}^{\alpha}\right)}=\frac{1}{\alpha}\left[\left(\gamma_{\alpha}^{\text {out }}(u), \gamma_{\alpha}^{\text {out }}(u)\right)_{L^{2}\left(\Gamma^{\alpha}\right)}-\left(\gamma_{\alpha}^{\text {inc }}(u), \gamma_{\alpha}^{\text {inc }}(u)\right)_{L^{2}\left(\Gamma^{\alpha}\right)}\right],
$$

where $(\cdot, \cdot)_{L^{2}\left(\mathcal{O}^{\alpha}\right)}$ and $(\cdot, \cdot)_{L^{2}\left(\Gamma^{\alpha}\right)}$ stand for the inner products associated with the norms of $L^{2}\left(\mathcal{O}^{\alpha}\right)$ and $L^{2}\left(\Gamma^{\alpha}\right)$.

In order to prove the existence of a solution of the problem (2.1), (2.2), (2.3), we proceed analogously to [4] and define the perturbed boundary operator

$$
\mathcal{B}_{\eta}=\mathcal{B} P+\frac{1}{1+\eta} Q, \quad \eta>0,
$$

as well as the operator $\mathcal{A}_{\eta}^{\alpha}=\mathcal{A}$ on the domain $H^{\alpha}\left(\mathcal{A}, \mathcal{B}_{\eta}\right)$, defined by:

$$
H^{\alpha}\left(\mathcal{A}, \mathcal{B}_{\eta}\right)=\left\{u \in H^{\alpha}(\mathcal{A}), \gamma_{\alpha}^{\text {out }}(u) \in L^{2}\left(\Gamma^{\alpha}\right), \gamma_{\alpha}^{\text {inc }}(u)=\mathcal{B}_{\eta} \gamma_{\alpha}^{\text {out }}(u)\right\} .
$$

For the perturbed problem, we have:

Lemma 3.3. For all $\eta>0$, for all $F_{\eta} \in H^{\alpha}\left(\mathcal{A}, \mathcal{B}_{\eta}\right)$, there exits a unique function $f_{\eta}^{\alpha} \in \mathcal{C}\left([0, T] ; H^{\alpha}\left(\mathcal{A}, \mathcal{B}_{\eta}\right)\right) \cap \mathcal{C}^{1}\left([0, T] ; L^{2}\left(\mathcal{O}^{\alpha}\right)\right)$, solving

$$
\left\{\begin{array}{l}
\alpha \partial_{t} f_{\eta}^{\alpha}+\mathcal{A} f_{\eta}^{\alpha}=0 \\
\left(f_{\eta}^{\alpha}\right)_{t=0}=F_{\eta}
\end{array}\right.
$$

Moreover, we have the following estimates:

$$
\left|f_{\eta}^{\alpha}\right|_{L^{2}\left(\mathcal{O}^{\alpha}\right)} \leq\left|F_{\eta}\right|_{L^{2}\left(\mathcal{O}^{\alpha}\right)} ; \quad\left|\alpha \partial_{t} f_{\eta}^{\alpha}\right|_{L^{2}\left(\mathcal{O}^{\alpha}\right)}=\left|\mathcal{A} f_{\eta}^{\alpha}\right|_{L^{2}\left(\mathcal{O}^{\alpha}\right)} \leq\left|\mathcal{A} F_{\eta}\right|_{L^{2}\left(\mathcal{O}^{\alpha}\right)}
$$


Lemma 3.4. Let $F_{I}$ be as in Hypothesis 2.1. There exits a sequence $(\eta)$ tending to zero and $\left(F_{\eta}\right)_{\eta>0}$ such that $F_{\eta} \in H^{\alpha}\left(\mathcal{A}, \mathcal{B}_{\eta}\right)$ and

$$
F_{\eta} \rightarrow F_{I}, \quad \mathcal{A} F_{\eta} \rightarrow \mathcal{A} F_{I} \text { weakly in } L^{2}\left(\mathcal{O}^{\alpha}\right) .
$$

The proofs of the above Lemmas are obtained in the same way as in the one dimensional case [4] and are skipped. Estimates (3.5) allow us to take the limit $\eta \rightarrow 0$ in the equation (3.4). In order to take the limit in the equality $\gamma_{\alpha}^{\text {inc }}\left(f_{\eta}^{\alpha}\right)=\mathcal{B}_{\eta} \gamma_{\alpha}^{\text {out }}\left(f_{\eta}^{\alpha}\right)$, we need to prove uniform estimates on traces. This is the aim of the next section.

\section{4. $L^{2}$ trace estimates}

In this section, we establish the control of $\gamma_{\alpha}^{\text {out }}(u), \gamma_{\alpha}^{\text {inc }}(u)$ in term of $|u|_{L^{2}\left(\mathcal{O}^{\alpha}\right)}$. The proofs are given in detail since the bidimensional geometry induces notable changes. Indeed, since the unit cell in the two dimensional case is a square and has a corner, singular terms appear as one uses straightforward extensions of the one dimensional proof. Therefore, we use cutoff functions which vanish at the square corners. Passing to the limit in the cutoff has then to be done carefully by linking the cutoff length to the parameter $\alpha$. Let us first define the cutoff function $\Phi_{\alpha, \beta}$ for $\alpha>0$ and $0<\beta<1$ by

$$
\Phi_{\alpha, \beta}(y)= \begin{cases}\frac{1}{\alpha \beta}(y-i \alpha), & y \in(i \alpha,(i+\beta) \alpha), \\ 1, & y \in(i+\beta) \alpha,(i+1-\beta) \alpha), \\ \frac{1}{\alpha \beta}((i+1) \alpha-y), & y \in((i+1-\beta) \alpha,(i+1) \alpha) .\end{cases}
$$

Proposition 4.1. Under Hypotheses 2.2 and 3.1, we have for all $u \in H^{\alpha}\left(\mathcal{A}, \mathcal{B}_{\eta}\right)$

i)

$$
\begin{gathered}
\left|P \gamma_{\alpha}^{\text {inc }}(u)\right|_{L^{2}\left(\Gamma^{\alpha}\right)}^{2} \leq\left|P \gamma_{\alpha}^{\text {out }}(u)\right|_{L^{2}\left(\Gamma^{\alpha}\right)}^{2} \leq \frac{2 \alpha}{1-K^{2}}(\mathcal{A} u, u)_{L^{2}\left(\mathcal{O}^{\alpha}\right)}, \\
\left(1-\frac{1}{(1+\eta)^{2}}\right)\left|Q \gamma_{\alpha}^{\text {out }}(u)\right|_{L^{2}\left(\Gamma^{\alpha}\right)}^{2} \leq 2 \alpha(\mathcal{A} u, u)_{L^{2}\left(\mathcal{O}^{\alpha}\right)} .
\end{gathered}
$$

ii)

$$
\begin{gathered}
(1+\eta) Q \gamma_{\alpha}^{\text {inc }}(u)=Q \gamma_{\alpha}^{\text {out }}(u) \\
\left|Q \gamma_{\alpha}^{\text {out }}(u) \sqrt{\Phi_{\alpha, \beta}}\right|_{L_{\delta}^{2}\left(\Gamma^{\alpha}\right)}^{2} \leq C_{1} \alpha|u|_{H^{\alpha}(\mathcal{A})}^{2}+C_{2}\left(1+\frac{\alpha}{\delta}+\frac{1}{\beta}\right)|u|_{L^{2}\left(\mathcal{O}^{\alpha}\right)}^{2},
\end{gathered}
$$

where $C_{1}, C_{2}$ are positive constants independent of $\alpha, \beta, \delta$ and $u$.

Proof: i) Let $u \in H^{\alpha}\left(\mathcal{A}, \mathcal{B}_{\eta}\right)$. By Green's formula (3.3), we have

$$
\begin{aligned}
2 \alpha & (\mathcal{A} u, u)_{L^{2}\left(\mathcal{O}^{\alpha}\right)}=\left|\gamma_{\alpha}^{\text {out }}(u)\right|_{L^{2}\left(\Gamma^{\alpha}\right)}^{2}-\left|\gamma_{\alpha}^{\text {inc }}(u)\right|_{L^{2}\left(\Gamma^{\alpha}\right)}^{2} \\
& =\left|\gamma_{\alpha}^{\text {out }}(u)\right|_{L^{2}\left(\Gamma^{\alpha}\right)}^{2}-\left|\mathcal{B}_{\eta} \gamma_{\alpha}^{\text {out }}(u)\right|_{L^{2}\left(\Gamma^{\alpha}\right)}^{2} \\
& =\left|P \gamma_{\alpha}^{\text {out }}(u)\right|_{L^{2}\left(\Gamma^{\alpha}\right)}^{2}-\left|\mathcal{B} P \gamma_{\alpha}^{\text {out }}(u)\right|_{L^{2}\left(\Gamma^{\alpha}\right)}^{2}+\left(1-\frac{1}{(1+\eta)^{2}}\right)\left|Q \gamma_{\alpha}^{\text {out }}(u)\right|_{L^{2}\left(\Gamma^{\alpha}\right)}^{2} \\
& \geq\left(1-K^{2}\right)\left|P \gamma_{\alpha}^{\text {out }}(u)\right|_{L^{2}\left(\Gamma^{\alpha}\right)}^{2}+\left(1-\frac{1}{(1+\eta)^{2}}\right)\left|Q \gamma_{\alpha}^{\text {out }}(u)\right|_{L^{2}\left(\Gamma^{\alpha}\right)}^{2} .
\end{aligned}
$$


which leads to (4.2), (4.3).

ii) Identity (4.4) is immediate. To prove (4.5), we only detail the contribution of $Q_{V}$ (the treatement of $Q_{H}$ being identical). Define $\psi_{1}\left(x_{1}, x_{2}\right)=\phi_{1}\left(x_{1}\right) \Phi_{\alpha, \beta}\left(x_{2}\right)$ where $\phi_{1}\left(x_{1}\right)=\frac{2}{\alpha}\left(x_{1}-\left(n+\frac{1}{2}\right) \alpha\right), \quad x_{1} \in(n \alpha,(n+1) \alpha)$. Using the Green's formula as before, we get for all $\delta>0$

$$
\begin{aligned}
2 & \left(\mathcal{A} u, u s^{\delta}\left(v_{1}\right) I^{\delta}\left(v_{2}\right) \psi_{1}\right)_{L^{2}\left(\mathcal{O}^{\alpha}\right)} \\
& +\int_{\mathcal{O}^{\alpha}}|u|^{2}\left[\omega_{1}^{\delta}(v) \partial_{x_{1}} \psi_{1}(x)+s^{\delta}\left(v_{1}\right) I^{\delta}\left(v_{2}\right) v_{2} \cdot \partial_{x_{2}} \psi_{1}(x)\right] d x d v \\
& +\int_{\mathcal{O}^{\alpha}}|u|^{2} \psi_{1}(x)\left[\partial_{x_{1}} V \partial_{v_{1}} s^{\delta}\left(v_{1}\right) I^{\delta}\left(v_{2}\right)+\partial_{x_{2}} V s^{\delta}\left(v_{1}\right) \partial_{v_{2}} I^{\delta}\left(v_{2}\right)\right] d x d v \\
& =\sum_{n \in \mathbb{Z}} \int_{\mathbb{R}} \int_{\mathbb{R}^{2}}\left[1+\frac{1}{(1+\eta)^{2}}\right]\left|Q_{V} \gamma_{V_{n}}^{\text {out }}(u)\right|^{2} \Phi_{\alpha, \beta}\left(x_{2}\right) \omega_{1}^{\delta}(v) d v d x_{2} \\
& +\sum_{n \in \mathbb{Z}} \int_{\mathbb{R}} \int_{\mathbb{R}^{2}}\left[\left|P_{V} \gamma_{V_{n}}^{\text {out }}(u)\right|^{2}+\left|P_{V} \gamma_{V_{n}}^{\text {inc }}(u)\right|^{2}\right] \Phi_{\alpha, \beta}\left(x_{2}\right) \omega_{1}^{\delta}(v) d v d x_{2} \\
& +2 \sum_{n \in \mathbb{Z}} \int_{\mathbb{R}} \int_{\mathbb{R}^{2}}\left[P_{V} \gamma_{V_{n}}^{\text {out }}(u)+\frac{1}{1+\eta} P_{V} \gamma_{V_{n}}^{\text {inc }}(u)\right] Q_{V} \gamma_{V_{n}}^{\text {out }}(u) \Phi_{\alpha, \beta}\left(x_{2}\right) \omega_{1}^{\delta}(v) d v d x_{2}
\end{aligned}
$$

The last term of the above identity can be bounded from below by

$$
\begin{aligned}
& -4 \sum_{n \in \mathbb{Z}} \int_{\mathbb{R}} \int_{\mathbb{R}^{2}}\left[\left|P_{V} \gamma_{V_{n}}^{\text {out }}(u)\right|^{2}+\left|P_{V} \gamma_{V_{n}}^{\text {inc }}(u)\right|^{2}\right] \Phi_{\alpha, \beta}\left(x_{2}\right) \omega_{1}^{\delta}(v) d v d x_{2} \\
& \quad-\frac{1}{4} \sum_{n \in \mathbb{Z}} \int_{\mathbb{R}} \int_{\mathbb{R}^{2}}\left[\left(1+\frac{1}{(1+\eta)^{2}}\right)\left|Q_{V} \gamma_{V_{n}}^{\text {out }}(u)\right|^{2}\right] \Phi_{\alpha, \beta}\left(x_{2}\right) \omega_{1}^{\delta}(v) d v d x_{2}
\end{aligned}
$$

Therefore, we get

$$
\begin{aligned}
& \left.\sum_{n \in \mathbb{Z}} \int_{\mathbb{R}} \int_{\mathbb{R}^{2}} \frac{3}{4}\left[1+\frac{1}{(1+\eta)^{2}}\right]\right)\left|Q_{V} \gamma_{V_{n}}^{\text {out }}(u)\right|^{2} \Phi_{\alpha, \beta}\left(x_{2}\right) \omega_{1}^{\delta}(v) d v d x_{2} \\
& \quad \leq 2\left(\mathcal{A} u, u s^{\delta}\left(v_{1}\right) I^{\delta}\left(v_{2}\right) \psi_{1}\right)_{L^{2}\left(\mathcal{O}^{\alpha}\right)}+\frac{1}{\alpha \beta} \int_{\mathcal{O}^{\alpha}}|u|^{2}\left[s^{\delta}\left(v_{1}\right) I^{\delta}\left(v_{2}\right) v_{2} \cdot \phi_{1}\left(x_{1}\right)\right] d x d v \\
& \quad+\frac{2}{\alpha} \int_{\mathcal{O}^{\alpha}}|u|^{2}\left[\omega_{1}^{\delta}(v) \Phi_{\alpha, \beta}\left(x_{2}\right)\right] d x d v \\
& \quad+\left|\nabla_{x} V\right|_{L^{\infty}}\left[\left|\partial_{v_{1}} s^{\delta}\left(v_{1}\right)\right|_{L^{\infty}}+\left|\partial_{v_{2}} I^{\delta}\left(v_{2}\right)\right|_{L^{\infty}}\right] \int_{\mathcal{O}^{\alpha}}|u|^{2} d x d v \\
& \quad+3 \sum_{n \in \mathbb{Z}} \int_{\mathbb{R}} \int_{\mathbb{R}^{2}}\left[\left|P_{V} \gamma_{V_{n}}^{\text {out }}(u)\right|^{2}+\left|P_{V} \gamma_{V_{n}}^{\text {inc }}(u)\right|^{2}\right]\left|v_{1}\right| d v d x_{2} .
\end{aligned}
$$

After multiplying (4.6) by $\alpha$ and using the boundedness of $\phi_{1}, \Phi_{\alpha, \beta}$, $\omega_{1}^{\delta}(v), s^{\delta}\left(v_{1}\right) I^{\delta}\left(v_{2}\right) v_{2}$ and the inequality $\delta\left|\partial_{v_{1}} s^{\delta}\left(v_{1}\right)\right|_{L^{\infty}}+\delta\left|\partial_{v_{2}} I^{\delta}\left(v_{2}\right)\right|_{L^{\infty}} \leq C$, we obtain

$$
\sum_{n \in \mathbb{Z}} \alpha\left|Q_{V} \gamma_{V_{n}}^{\text {out }}(u) \sqrt{\Phi_{\alpha, \beta}}\right|_{L^{2}\left(\mathbb{R}_{x_{2}}, L_{\delta, 1}^{2}\left(\mathbb{R}^{2}\right)\right)}^{2} \leq C_{1} \alpha|u|_{H^{\alpha}(\mathcal{A})}^{2}+C_{2}\left(1+\frac{\alpha}{\delta}+\frac{1}{\beta}\right)|u|_{L^{2}\left(\mathcal{O}^{\alpha}\right)}^{2},
$$

Proceeding analogously we obtain the same inequality of $Q_{V} \gamma_{H_{m}}^{\text {out }}(u)$ which leads to (4.5). 
4.1. Existence of a solution to the problem. We can prove the first part of the Theorem 2.1. More precisely, we have

Proposition 4.2. Under hypotheses 2.1, 2.2, 2.3, 3.1, there exists a solution $f^{\alpha}$ to problem (2.1), (2.2), (2.3), such that $f^{\alpha} \in L^{\infty}\left(0, T ; L^{2}\left(\mathcal{O}^{\alpha}\right)\right), \mathcal{A} f^{\alpha} \in L^{\infty}\left(0, T ; L^{2}\left(\mathcal{O}^{\alpha}\right)\right)$, $P \gamma_{\alpha}^{\text {out }}\left(f^{\alpha}\right) \in L^{\infty}\left(0, T ; L^{2}\left(\Gamma^{\alpha}\right)\right), Q \gamma_{\alpha}^{\text {out }}\left(f^{\alpha}\right) \sqrt{\Phi_{\alpha, \beta}} \in L^{\infty}\left(0, T ; L_{\delta}^{2}\left(\Gamma^{\alpha}\right)\right)$, for all $\delta>0$ and $0<\beta<1$, and the boundary condition is satisfied in the following sense:

$$
\begin{gathered}
P \gamma_{\alpha}^{\text {inc }}\left(f^{\alpha}\right)=\mathcal{B P} \gamma_{\alpha}^{\text {out }}\left(f^{\alpha}\right), \\
Q \gamma_{\alpha}^{\text {inc }}\left(f^{\alpha}\right)=Q \gamma_{\alpha}^{\text {out }}\left(f^{\alpha}\right) .
\end{gathered}
$$

Lemma 4.1. There exists a constant $C$ independent of $\alpha$ such that

$$
\int_{0}^{T}\left|P \gamma_{\alpha}^{i n c}\left(f_{\eta}^{\alpha}\right)(t)\right|_{L^{2}\left(\Gamma^{\alpha}\right)}^{2} d t \leq C \alpha^{2}\left|F_{I}\right|_{L^{2}\left(\mathbb{R}^{2} \times \mathbb{R}\right)} .
$$

The proof of Proposition 4.2 and Lemma 4.1 follow exactly the same lines as those Proposition 3.11 and Lemma 3.13 of [4]. They are skipped.

\section{Convergence towards the macroscopic model}

In this section, we prove the convergence part of Theorem 2.1, according to the scheme outlined in the introduction.

5.1. $L^{2}$ estimates. Let us first summarize the $L^{2}$ estimates deduced from the previous section.

Lemma 5.1. The solution $f^{\alpha}$ of problem constructed in the previous section satisfies

$$
\begin{gathered}
\left|f^{\alpha}\right|_{C^{0}\left([0, T], L^{2}\left(\mathcal{O}^{\alpha}\right)\right)} \leq\left|F_{I}\right|_{L^{2}\left(\mathbb{R}^{2} \times \mathbb{R}_{+}^{2}\right)} \\
\int_{0}^{T}\left|P \gamma_{\alpha}^{\text {out }}\left(f^{\alpha}\right)(t)\right|_{L^{2}\left(\Gamma^{\alpha}\right)}^{2} d t \leq C \alpha^{2}\left|F_{I}\right|_{L^{2}\left(\mathbb{R}^{2} \times \mathbb{R}_{+}^{2}\right)}^{2} \\
\int_{0}^{T}\left|P \gamma_{\alpha}^{\text {inc }}\left(f^{\alpha}\right)(t)\right|_{L^{2}\left(\Gamma^{\alpha}\right)}^{2} d t \leq C \alpha^{2}\left|F_{I}\right|_{L^{2}\left(\mathbb{R}^{2} \times \mathbb{R}_{+}^{2}\right)}^{2} \\
\int_{0}^{T}\left|Q \gamma_{\alpha}^{\text {out }}\left(f^{\alpha}\right)(t) \sqrt{\Phi_{\alpha, \beta}}\right|_{L_{\delta}^{2}\left(\Gamma^{\alpha}\right)}^{2} d t \leq\left(C_{\delta}+\frac{C}{\beta}\right)\left|F_{I}\right|_{H^{\alpha}(\mathcal{A})},
\end{gathered}
$$

where $C$ denotes generic constants independent of $\alpha$ and of the data and $\Phi_{\alpha, \beta}$ is defined in (4.1).

We immediately deduce from the Lemma 5.1 the existence of a subsequence (still denoted by $\left.f^{\alpha}\right)$ and a function $f^{0}$ in $L^{\infty}\left([0, T], L^{2}\left(\mathbb{R}^{2} \times \mathbb{R}^{2}\right)\right)$ such that

$$
f^{\alpha} \rightarrow f^{0} \text { in } L^{\infty}\left((0, T), L^{2}\left(\mathbb{R}^{2} \times \mathbb{R}^{2}\right)\right) \text { weak star },
$$

when $\alpha \rightarrow 0$. In view of Lemma 5.1, the trace $\gamma_{\alpha}^{\text {out }}\left(f^{\alpha}\right)$ and $\gamma_{\alpha}^{\text {inc }}\left(f^{\alpha}\right)$ converge to functions of $\left(\varepsilon_{1}, \varepsilon_{2}\right)$ when $\varepsilon_{i}=\frac{v_{i}^{2}}{2}$. In the next Lemma, we prove that this asymptotic behavior holds for the whole function $f^{0}$. 
Lemma 5.2. Let $f^{0}$ be the limit of the sequence $f^{\alpha}$. Then, there exists a function $F\left(t, x, \varepsilon_{1}, \varepsilon_{2}\right)$ such that

$$
f^{0}(t, x, v)=F\left(t, x, \frac{v_{1}^{2}}{2}, \frac{v_{2}^{2}}{2}\right)
$$

Proof: Setting $\beta=\sqrt{\alpha}$, we notice the $\Phi_{\alpha, \sqrt{\alpha}} \in L_{\text {loc }}^{2}(\mathbb{R})$ and $\Phi_{\alpha, \sqrt{\alpha}} \rightarrow 1$ in the strong topology of $L_{l o c}^{2}(\mathbb{R})$ as $\alpha \rightarrow 0$. Let us first prove that $f^{0}(t, x, v)$ is even with respect to $v_{1}$. To this aim, we consider a test function $\varphi$ in $\mathcal{D}\left((0, T) \times \mathbb{R}^{2} \times \mathbb{R}^{2}\right)$ such that $\varphi$ is even with respect to $v_{1}$ and prove that

$$
\left\langle\left(f^{\alpha} v_{1}, \varphi\right\rangle_{\mathcal{D}^{\prime}, \mathcal{D}}=0\right.
$$

The starting point is the decomposition

$$
\left\langle\left(f^{\alpha} v_{1}, \varphi\right\rangle_{\mathcal{D}^{\prime}, \mathcal{D}}=\left\langle\left(f^{\alpha} v_{1}\left(1-\Phi_{\alpha, \sqrt{\alpha}}\right), \varphi\right\rangle_{\mathcal{D}^{\prime}, \mathcal{D}}+\left\langle\left(f^{\alpha} v_{1} \Phi_{\alpha, \sqrt{\alpha}}, \varphi\right\rangle_{\mathcal{D}^{\prime}, \mathcal{D}}\right.\right.\right.
$$

We shall now prove the convergence of both terms of the right hand side to zero as $\alpha$ tends to zero. Let us start with the second term. We first introduce the function

$$
\psi^{\alpha}\left(x_{1}\right)=\left(n+\frac{1}{2}\right) \alpha-x_{1} ; \text { on }[n \alpha,(n+1) \alpha] .
$$

Applying the Green's formula (3.3) with $\varphi \psi^{\alpha} \Phi_{\alpha, \sqrt{\alpha}}$ as a test function leads to

$$
\begin{aligned}
& -\int_{0}^{T} \int_{\mathcal{O}^{\alpha}} v_{1} f^{\alpha} \varphi \partial_{x_{1}} \psi^{\alpha}\left(x_{1}\right) \Phi_{\alpha, \sqrt{\alpha}}\left(x_{2}\right) d x d v d t \\
& =\int_{0}^{T} \int_{\mathcal{O}^{\alpha}} f^{\alpha}\left(\alpha \partial_{t} \varphi+\mathcal{A}^{\alpha} \varphi\right) \psi^{\alpha}\left(x_{1}\right) \Phi_{\alpha, \sqrt{\alpha}}\left(x_{2}\right) d x d v d t \\
& \quad+\int_{0}^{T} \int_{\mathcal{O}^{\alpha}} v_{2} f^{\alpha} \varphi \psi^{\alpha}\left(x_{1}\right) \partial_{x_{2}} \Phi_{\alpha, \sqrt{\alpha}}\left(x_{2}\right) d x d v d t \\
& \quad-\int_{0}^{T} \int_{\mathbb{R}^{2}} \sum_{n \in \mathbb{Z}} \int_{\mathbb{R}} v_{1}\left\{\gamma_{V_{n+1}}^{-}\left(f^{\alpha} \varphi \psi^{\alpha}\right)-\gamma_{V_{n}}^{+}\left(f^{\alpha} \varphi \psi^{\alpha}\right)\right\} \Phi_{\alpha, \sqrt{\alpha}}\left(x_{2}\right) d x_{2} d v d t .
\end{aligned}
$$

Since $\left|\psi^{\alpha}\right|_{L^{\infty}} \leq \alpha$ and $\left|\Phi_{\alpha, \sqrt{\alpha}}\right|_{L^{\infty}} \leq 1$, the first term of the right hand side of (5.5), denoted by $M^{\alpha}$, can be bounded by

$$
\left|M^{\alpha}\right| \leq \alpha N(\varphi)\left|f^{\alpha}\right|_{L^{2}\left([0, T] \times \mathbb{R}^{2} \times \mathbb{R}^{2}\right)},
$$

where $N(\varphi)$ depends on $\varphi$ and its derivatives. Since $\partial_{x_{1}} \psi^{\alpha}=-1$ and $\gamma_{V_{n+1}}^{-}(\psi)^{\alpha}=-\frac{\alpha}{2}$, $\gamma_{V_{n}}^{+}(\psi)^{\alpha}=\frac{\alpha}{2}$, we deduce from (5.5) that

$$
\begin{aligned}
\left\langle f^{\alpha} v_{1} \Phi_{\alpha, \sqrt{\alpha}}, \varphi\right\rangle_{\mathcal{D}^{\prime}, \mathcal{D}}=M^{\alpha}+\int_{0}^{T} \int_{\mathcal{O}^{\alpha}} v_{2} f^{\alpha} \varphi \psi^{\alpha}\left(x_{1}\right) \partial_{x_{2}} \Phi_{\alpha, \sqrt{\alpha}}\left(x_{2}\right) d x d v d t \\
+\frac{\alpha}{2} \int_{0}^{T} \int_{\mathbb{R}^{2}} \sum_{n \in \mathbb{Z}} \int_{\mathbb{R}} v_{1}\left\{\gamma_{V_{n+1}}^{-}\left(f^{\alpha} \varphi\right)+\gamma_{V_{n}}^{+}\left(f^{\alpha} \varphi\right)\right\} \Phi_{\alpha, \sqrt{\alpha}}\left(x_{2}\right) d x_{2} d v d t .
\end{aligned}
$$

Using the orthogonality of $Q_{V} \gamma_{V_{n}}^{+}\left(f^{\alpha}\right)$ (respectively $Q_{V} \gamma_{V_{n+1}}^{-}\left(f^{\alpha}\right)$ ) and $v_{1} \gamma_{V_{n}}^{+}(\varphi)$ (respectively $v_{1} \gamma_{V_{n+1}}^{-}(\varphi)$ ), we obtain

$$
\begin{aligned}
\left\langle f^{\alpha} v_{1} \Phi_{\alpha, \sqrt{\alpha}}, \varphi\right\rangle_{\mathcal{D}^{\prime}, \mathcal{D}}=M^{\alpha}+\int_{0}^{T} \int_{\mathcal{O}^{\alpha}} v_{2} f^{\alpha} \varphi \psi^{\alpha}\left(x_{1}\right) \partial_{x_{2}} \Phi_{\alpha, \sqrt{\alpha}}\left(x_{2}\right) d x d v d t \\
+\frac{\alpha}{2} \int_{0}^{T} \int_{\mathbb{R}^{2}} \sum_{n \in \mathbb{Z}} \int_{\mathbb{R}} v_{1} \Phi_{\alpha, \sqrt{\alpha}}\left(x_{2}\right)\left\{P_{V} \gamma_{V_{n}}^{+}\left(f^{\alpha}\right)\left(t, x_{2}, v\right) \varphi\left(t, n \alpha, x_{2}, v\right)\right. \\
\left.+P_{V} \gamma_{V_{n+1}}^{-}\left(f^{\alpha}\right)\left(t, x_{2}, v\right) \varphi\left(t,(n+1) \alpha, x_{2}, v\right)\right\} d x_{2} d v d t
\end{aligned}
$$


Since $\left|\Phi_{\alpha, \sqrt{\alpha}}\right|_{L^{\infty}} \leq 1$ and $\varphi$ is compactly supported, we have

$$
\begin{aligned}
& \left|\int_{0}^{T} \int_{\mathbb{R}^{2}} \sum_{n \in \mathbb{Z}} \frac{\alpha}{2} \int_{\mathbb{R}} v_{1} P_{V} \gamma_{V_{n+1}}^{-}\left(f^{\alpha}\right)\left(t, x_{2}, v\right) \Phi_{\alpha, \sqrt{\alpha}}\left(x_{2}\right) \varphi\left(t,(n+1) \alpha, x_{2}, v\right) d x_{2} d v d t\right| \\
& \leq N(\varphi)\left[\left|P \gamma_{\alpha}^{\text {out }}\left(f^{\alpha}\right)(t)\right|_{L^{2}\left([0, T], L^{2}\left(\Gamma^{\alpha}\right)\right)}^{2}+\left|P \gamma_{\alpha}^{\text {inc }}\left(f^{\alpha}\right)(t)\right|_{L^{2}\left([0, T], L^{2}\left(\Gamma^{\alpha}\right)\right)}^{2}\right]^{\frac{1}{2}}=O(\alpha) .
\end{aligned}
$$

Besides,

$$
\begin{aligned}
& \int_{0}^{T} \int_{\mathcal{O}^{\alpha}} v_{2} f^{\alpha} \varphi \psi^{\alpha}\left(x_{1}\right) \partial_{x_{2}} \Phi_{\alpha, \sqrt{\alpha}}\left(x_{2}\right) d x d v d t \\
= & \frac{1}{\alpha \sqrt{\alpha}} \int_{0}^{T} \int_{\mathbb{R}^{2}} \int_{\mathbb{R}} \sum_{m \in \mathbb{Z}} \int_{m \alpha}^{(m+\sqrt{\alpha}) \alpha} v_{1} f^{\alpha}(t, x, v) \psi^{\alpha}\left(x_{1}\right) \varphi(t, x, v) d x_{2} d v d t \\
& -\frac{1}{\alpha \sqrt{\alpha}} \int_{0}^{T} \int_{\mathbb{R}^{2}} \int_{\mathbb{R}} \sum_{m \in \mathbb{Z}} \int_{(m+1-\sqrt{\alpha}) \alpha}^{(m+1) \alpha} v_{1} f^{\alpha}(t, x, v) \psi^{\alpha}\left(x_{1}\right) \varphi(t, x, v) d x_{2} d v d t \\
= & \frac{1}{\alpha \sqrt{\alpha}} \int_{0}^{T} \int_{\mathbb{R}^{2}} \int_{\mathbb{R}} \sum_{m \in \mathbb{Z}} \int_{m \alpha}^{(m+\sqrt{\alpha}) \alpha} v_{1} f^{\alpha}(t, x, v) \psi^{\alpha}\left(x_{1}\right)[\varphi(t, x, v) \\
& \left.-\varphi\left(t, x_{1},\left(m+\frac{1}{2}\right) \alpha, v\right)\right] d x_{2} d v d t \\
& +\frac{1}{\alpha \sqrt{\alpha}} \int_{0}^{T} \int_{\mathbb{R}^{2}} \int_{\mathbb{R}} \sum_{m \in \mathbb{Z}} \int_{(m+1) \alpha}^{(m+1) \alpha} v_{1} f^{\alpha}(t, x, v) \psi^{\alpha}\left(x_{1}\right)\left[\varphi\left(t, x_{1},\left(m+\frac{1}{2}\right) \alpha, v\right)\right. \\
& -\varphi(t, x, v)] d x_{2} d v d t .
\end{aligned}
$$

Using the inequalities

$$
\begin{aligned}
& \int_{0}^{T} \int_{\mathbb{R}^{2}} \int_{\mathbb{R}} \sum_{m \in \mathbb{Z}} \int_{(m+1-\sqrt{\alpha}) \alpha}^{(m+1) \alpha}\left(\varphi\left(t, x_{1},\left(m+\frac{1}{2}\right) \alpha, \varepsilon\right)-\varphi\left(t, x_{1}, x_{2}, v\right)\right)^{2} d x_{1} d x_{2} d v d t \\
& \quad \leq C(\varphi) \alpha^{2} \\
& \int_{0}^{T} \int_{\mathbb{R}^{2}} \int_{\mathbb{R}} \sum_{m \in \mathbb{Z}} \int_{m \alpha}^{(m+\sqrt{\alpha}) \alpha}\left(\varphi\left(t, x_{1},\left(m+\frac{1}{2}\right) \alpha, v\right)-\varphi\left(t, x_{1}, x_{2}, v\right)\right)^{2} d x_{1} d x_{2} d v d t . \\
& \quad \leq C(\varphi) \alpha^{2}
\end{aligned}
$$

and $\left|\psi^{\alpha}\right|_{L^{\infty}} \leq \alpha$, we obtain

$$
\int_{0}^{T} \int_{\mathcal{O}^{\alpha}} v_{2} f^{\alpha} \varphi \psi^{\alpha}\left(x_{1}\right) \partial_{x_{2}} \Phi_{\alpha, \sqrt{\alpha}}\left(x_{2}\right) d x d v d t \leq \sqrt{\alpha} N(\varphi)\left|f^{\alpha}\right|_{L^{2}\left([0, T] \times \mathbb{R}^{2} \times \mathbb{R}^{2}\right)} .
$$

Therefore, in view of (5.6),(5.8) and (5.9), (5.7) leads to the estimates

$$
\left\langle f^{\alpha} v_{1} \Phi_{\alpha, \sqrt{\alpha}}, \varphi\right\rangle_{\mathcal{D}^{\prime}, \mathcal{D}}=O(\alpha)
$$

Besides

$$
\left\langle f^{\alpha} v_{1}\left(1-\Phi_{\alpha, \sqrt{\alpha}}\right), \varphi\right\rangle_{\mathcal{D}^{\prime}, \mathcal{D}} \leq\left|f^{\alpha}\right|_{L^{2}\left([0, T] \times \mathbb{R}^{2} \times \mathbb{R}^{2}\right)} \mid v_{1}\left(1-\left.\Phi_{\alpha \sqrt{\alpha})}\right|_{L^{2}\left([0, T] \times \mathbb{R}^{2} \times \mathbb{R}^{2}\right)} .\right.
$$

Since $\varphi$ is compactly supported, $\left|\Phi_{\alpha, \sqrt{\alpha}}\right|_{L^{\infty}} \leq 1$ and $\Phi_{\alpha, \sqrt{\alpha}}$ tends to 1 a.e., the Lebesgue dominated convergence theorem insures the convergence to zero of $\mid v_{1}(1-$ $\left.\Phi_{\alpha \sqrt{\alpha})}\right|_{L^{2}\left([0, T] \times \mathbb{R}^{2} \times \mathbb{R}^{2}\right)}$. We have finally proven that

$$
\lim _{\alpha \rightarrow 0^{+}}\left\langle v_{1} f^{\alpha}, \varphi\right\rangle_{\mathcal{D}^{\prime}, \mathcal{D}}=0
$$


which shows that

$$
\left\langle v_{1} f^{0}, \varphi\right\rangle_{\mathcal{D}^{\prime}, \mathcal{D}}=0
$$

Therefore, $f^{0}$ is even with respect to $v_{1}$. Similarly, it is even with respect to $v_{2}$.

5.2. The kinetic problem in weak form and the continuity equation. We first write problem (2.1), (2.2), (2.3) in a weak form. Green's formula (3.3) immediately gives the following lemmas:

Lemma 5.3. Let $f^{\alpha}$ be the solution of (2.1), (2.2), (2.3). For any $\phi \in C_{c}^{1}\left([0, T], H_{0}^{\alpha}(\mathcal{A})\right)$ such that $\phi(T, \cdot, \cdot, \cdot)=0$, we have

$$
\begin{aligned}
& \int_{0}^{T} \int_{\mathcal{O}^{\alpha}} f^{\alpha}(t, x, v)\left[\alpha \partial_{t} \phi+v \cdot \nabla_{x} \phi+\nabla_{x} V \cdot \nabla_{v} \phi\right](t, x, v) d x d v d t \\
& +\alpha \int_{\mathcal{O}^{\alpha}} f_{I}^{\alpha}(x, v) \phi(0, x, v) d x d v=-\frac{1}{\alpha} \int_{0}^{T}\left(\gamma_{\alpha}^{\text {out }}\left(f^{\alpha}\right), \gamma_{\alpha}^{\text {out }}(\phi)-\mathcal{B} \gamma_{\alpha}^{\text {inc }}(\phi)\right)_{L^{2}\left(\Gamma^{\alpha}\right)} .
\end{aligned}
$$

Let us define the "macroscopic" quantities

$$
\begin{gathered}
F^{\alpha}\left(t, x, \varepsilon_{1}, \varepsilon_{2}\right)=\frac{1}{4} \sum_{s_{1}, s_{2}=\mp 1} f^{\alpha}\left(t, x, s_{1} \sqrt{2 \varepsilon_{1}}, s_{2} \sqrt{2 \varepsilon_{2}}\right), \\
J_{1}^{\alpha}\left(t, x, \varepsilon_{1}, \varepsilon_{2}\right)=\frac{1}{\alpha \sqrt{2 \varepsilon_{2}}} \sum_{s_{2}=\mp 1}\left[f^{\alpha}\left(t, x, \sqrt{2 \varepsilon_{1}}, s_{2} \sqrt{2 \varepsilon_{2}}\right)-f^{\alpha}\left(t, x,-\sqrt{2 \varepsilon_{1}}, s_{2} \sqrt{2 \varepsilon_{2}}\right)\right] \\
J_{2}^{\alpha}\left(t, x, \varepsilon_{1}, \varepsilon_{2}\right)=\frac{1}{\alpha \sqrt{2 \varepsilon_{1}}} \sum_{s_{1}=\mp 1}\left[f^{\alpha}\left(t, x, s_{1} \sqrt{2 \varepsilon_{1}}, \sqrt{2 \varepsilon_{2}}\right)-f^{\alpha}\left(t, x, s_{1} \sqrt{2 \varepsilon_{1}},-\sqrt{2 \varepsilon_{2}}\right)\right] .
\end{gathered}
$$

We have

Lemma 5.4. Let $f^{\alpha}$ be the solution of (2.1), (2.2), (2.3). For any test function $\phi \in C_{c}^{1}\left([0, T] \times \mathbb{R}^{2} \times \mathbb{R}_{+}^{2}\right)$ such that $\phi(T, \cdot, \cdot, \cdot)=0$, we have

$$
\begin{aligned}
& \int_{0}^{T} \int_{\Omega^{\alpha} \times \mathbb{R}_{+}^{2}} \frac{2}{\sqrt{\varepsilon_{1} \varepsilon_{2}}} F^{\alpha}\left(t, x, \varepsilon_{1}, \varepsilon_{2}\right) \partial_{t} \phi\left(t, x, \varepsilon_{1}, \varepsilon_{2}\right) d x d \varepsilon_{1} d \varepsilon_{2} d t \\
& +\int_{0}^{T} \int_{\Omega^{\alpha} \times \mathbb{R}_{+}^{2}} J_{1}^{\alpha}\left(t, x, \varepsilon_{1}, \varepsilon_{2}\right)\left(\partial_{x_{1}}+\partial_{x_{1}} V \partial_{\varepsilon_{1}}\right) \phi\left(t, x, \varepsilon_{1}, \varepsilon_{2}\right) d x d \varepsilon_{1} d \varepsilon_{2} d t \\
& \left.+\int_{0}^{T} \int_{\Omega^{\alpha} \times \mathbb{R}_{+}^{2}} J_{2}^{\alpha}\left(t, x, \varepsilon_{1}, \varepsilon_{2}\right)\left(\partial_{x_{2}}+\partial_{x_{2}} V \partial_{\varepsilon_{2}}\right) \phi\left(t, x, \varepsilon_{1}, \varepsilon_{2}\right)\right) d x d \varepsilon_{1} d \varepsilon_{2} d t \\
& +\int_{\mathbb{R}^{2} \times \mathbb{R}_{+}^{2}} \frac{2}{\sqrt{\varepsilon_{1} \varepsilon_{2}}} F_{I}\left(x, \varepsilon_{1}, \varepsilon_{2}\right) \phi\left(0, x, \varepsilon_{1}, \varepsilon_{2}\right) d x d \varepsilon_{1} d \varepsilon_{2}=0 .
\end{aligned}
$$
obtain

Proof: Let $f_{\eta}^{\alpha}$ be the solution of (3.4). From the Green's formula (3.3), we

$$
\begin{aligned}
& \int_{0}^{T} \int_{\mathcal{O}^{\alpha}} f_{\eta}^{\alpha}(t, x, v)\left[\alpha \partial_{t} \phi+v \cdot \nabla_{x} \phi+\nabla_{x} V \cdot \nabla_{v} \phi\right](t, x, v) d x d v d t \\
& \quad+\alpha \int_{\mathcal{O}^{\alpha}}^{T} F_{\eta}(x, v) \phi(0, x, v) d x d v \\
& \quad=\frac{1}{\alpha} \int_{0}^{T}\left(\gamma_{\alpha}^{\text {out }}\left(f_{\eta}^{\alpha}\right), \gamma_{\alpha}^{\text {out }}(\phi)\right)_{L^{2}\left(\Gamma^{\alpha}\right)}-\frac{1}{\alpha} \int_{0}^{T}\left(\gamma_{\alpha}^{\text {inc }}\left(f_{\eta}^{\alpha}\right), \gamma_{\alpha}^{\text {inc }}(\phi)\right)_{L^{2}\left(\Gamma^{\alpha}\right)},
\end{aligned}
$$


where $\gamma(\phi)=\gamma_{\alpha}^{\text {out }}(\phi)=\gamma_{\alpha}^{\text {inc }}(\phi)$. Thanks to the co-area formula, and to the orthogonality between $P \gamma_{\alpha}^{\text {out }}\left(f_{\eta}^{\alpha}\right), P \gamma_{\alpha}^{\text {inc }}\left(f_{\eta}^{\alpha}\right)$ and $\gamma(\phi)$, we have:

$$
\begin{aligned}
& \int_{0}^{T} \int_{\Omega^{\alpha} \times \mathbb{R}_{+}^{2}} \frac{2}{\sqrt{\varepsilon_{1} \varepsilon_{2}}} F_{\eta}^{\alpha}\left(t, x, \varepsilon_{1}, \varepsilon_{2}\right) \alpha \partial_{t} \phi\left(t, x, \varepsilon_{1}, \varepsilon_{2}\right) d x d \varepsilon_{1} d \varepsilon_{2} d t \\
& +\alpha \int_{0}^{T} \int_{\Omega^{\alpha} \times \mathbb{R}_{+}^{2}} J_{1}^{\alpha}\left(t, x, \varepsilon_{1}, \varepsilon_{2}\right)\left(\partial_{x_{1}}+\partial_{x_{1}} V \partial_{\varepsilon_{1}}\right) \phi\left(t, x, \varepsilon_{1}, \varepsilon_{2}\right) d x d \varepsilon_{1} d \varepsilon_{2} d t \\
& +\alpha \int_{0}^{T} \int_{\Omega^{\alpha} \times \mathbb{R}_{+}^{2}} J_{2}^{\alpha}\left(t, x, \varepsilon_{1}, \varepsilon_{2}\right)\left(\partial_{x_{2}}+\partial_{x_{2}} V \partial_{\varepsilon_{2}}\right) \phi\left(t, x, \varepsilon_{1}, \varepsilon_{2}\right) d x d \varepsilon_{1} d \varepsilon_{2} d t \\
& +\alpha \int_{\mathbb{R}^{2} \times \mathbb{R}_{+}^{2}} \frac{2}{\sqrt{\varepsilon_{1} \varepsilon_{2}}} F_{I}\left(x, \varepsilon_{1}, \varepsilon_{2}\right) \phi\left(0, x, \varepsilon_{1}, \varepsilon_{2}\right) d x d \varepsilon_{1} d \varepsilon_{2} \\
& \left.=\frac{1}{\alpha} \int_{0}^{T}\left(1-\frac{1}{1+\eta}\right) Q \gamma_{\alpha}^{\text {out }}\left(f_{\eta}^{\alpha}\right), \gamma(\phi)\right)_{L^{2}\left(\Gamma^{\alpha}\right)} .
\end{aligned}
$$

(5.14) is then obtained by letting $\eta$ tend to zero.

We are now aiming at taking the limit $\alpha \rightarrow 0$. We need to pass to the limit in $F^{\alpha}$ and in $\left(J^{\alpha}=\left(J_{1}^{\alpha}, J_{2}^{\alpha}\right)^{T}\right)$. It is readily seen that

$\frac{2}{\sqrt{\varepsilon_{1} \varepsilon_{2}}} F^{\alpha}\left(t, x, \varepsilon_{1}, \varepsilon_{2}\right) \rightarrow \frac{1}{4} \sum_{s_{1}, s_{2}=\mp 1} f^{0}\left(t, x, s_{1} \sqrt{2 \varepsilon_{1}}, s_{2} \sqrt{2 \varepsilon_{2}}\right) \frac{2}{\sqrt{\varepsilon_{1} \varepsilon_{2}}}=\frac{2}{\sqrt{\varepsilon_{1} \varepsilon_{2}}} F\left(t, x, \varepsilon_{1}, \varepsilon_{2}\right)$

in $L^{\infty}\left([0, T], L^{2}\left(\mathbb{R}^{2} \times \mathbb{R}_{+}^{2}\right)\right)$ weak star. The analysis of the subsequence $J^{\alpha}=\left(J_{1}^{\alpha}, J_{2}^{\alpha}\right)^{T}$ is done in the next subsection.

5.3. Existence of a limit for the current. The function $J^{\alpha}=\left(J_{1}^{\alpha}, J_{2}^{\alpha}\right)^{T}$ is defined on $\left(\Omega^{\alpha} \times \mathbb{R}_{+}^{2}\right)^{2}$ and has traces $\gamma_{V_{n}}^{\mp}\left(J_{1}^{\alpha}\right), \gamma_{H_{m}}^{\mp}\left(J_{2}^{\alpha}\right)$ at the interfaces, obviously given by

$$
\begin{aligned}
& \gamma_{V_{n}}^{\mp}\left(J_{1}^{\alpha}\right)\left(t, x_{2}, \varepsilon_{1}, \varepsilon_{2}\right)=\frac{1}{\alpha \sqrt{2 \varepsilon_{2}}} \sum_{s_{2}=\mp 1}\left[\gamma_{V_{n}}^{\mp}\left(f^{\alpha}\right)\left(t, x_{2}, \sqrt{2 \varepsilon_{1}}, s_{2} \sqrt{2 \varepsilon_{2}}\right)\right. \\
& \left.-\gamma_{V_{n}}^{\mp}\left(f^{\alpha}\right)\left(t, x_{2},-\sqrt{2 \varepsilon_{1}}, s_{2} \sqrt{2 \varepsilon_{2}}\right)\right] \\
& \gamma_{H_{m}}^{\mp}\left(J_{2}^{\alpha}\right)\left(t, x_{1}, \varepsilon_{1}, \varepsilon_{2}\right)=\frac{1}{\alpha \sqrt{2 \varepsilon_{1}}} \sum_{s_{1}=\mp 1}\left[\gamma_{H_{m}}^{\mp}\left(f^{\alpha}\right)\left(t, x_{1}, s_{1} \sqrt{2 \varepsilon_{1}}, \sqrt{2 \varepsilon_{2}}\right)\right. \\
& \left.-\gamma_{H_{m}}^{\mp}\left(f^{\alpha}\right)\left(t, x_{1}, s_{1} \sqrt{2 \varepsilon_{1}},-\sqrt{2 \varepsilon_{2}}\right)\right] \text {. }
\end{aligned}
$$

Let us construct the piecewise constant functions

$$
\begin{aligned}
& \tilde{J}_{V}^{\alpha}\left(t, x_{1}, x_{2}, \varepsilon_{1}, \varepsilon_{2}\right)=\frac{1}{2}\left(\gamma_{V_{n}}^{-}\left(J_{1}^{\alpha}\right)+\gamma_{V_{n}}^{+}\left(J_{1}^{\alpha}\right)\right)\left(t, x_{2}, \varepsilon_{1}, \varepsilon_{2}\right), \\
& x_{1} \in\left(\left(n-\frac{1}{2}\right) \alpha,\left(n+\frac{1}{2}\right) \alpha\right), x_{2} \in \mathbb{R}, \\
& \tilde{J}_{H}^{\alpha}\left(t, x_{1}, x_{2}, \varepsilon_{1}, \varepsilon_{2}\right)=\frac{1}{2}\left(\gamma_{H_{m}}^{-}\left(J_{2}^{\alpha}\right)+\gamma_{H_{m}}^{+}\left(J_{2}^{\alpha}\right)\right)\left(t, x_{1}, \varepsilon_{1}, \varepsilon_{2}\right), \\
& x_{1} \in \mathbb{R}, x_{2} \in\left(\left(m-\frac{1}{2}\right) \alpha,\left(m+\frac{1}{2}\right) \alpha\right) \text {. }
\end{aligned}
$$

The existence of a limit of $J^{\alpha}$ is obtained through that of $\tilde{J}^{\alpha}=\left(\tilde{J}_{V}^{\alpha}, \tilde{J}_{H}^{\alpha}\right)$ as the following lemma states: 
Lemma 5.5. The function $\tilde{J}^{\alpha}=\left(\tilde{J}_{V}^{\alpha}, \tilde{J}_{H}^{\alpha}\right)$ is bounded in $L^{2}\left([0, T] \times \mathbb{R}^{2}, L_{l o c}^{2}\left(\mathbb{R}_{+}^{2}\right)\right)^{2}$ and we have

$$
\begin{aligned}
& J_{1}^{\alpha}-\tilde{J}_{V}^{\alpha} \rightarrow 0, \\
& J_{2}^{\alpha}-\tilde{J}_{H}^{\alpha} \rightarrow 0,
\end{aligned}
$$

as $\alpha \rightarrow 0$, in the distributional sense. There exists $J=\left(J_{1}, J_{2}\right)$ in $L^{2}\left([0, T] \times \mathbb{R}^{2} \times\right.$ $\left.\mathbb{R}_{+}^{2}\right)^{2}$ such that up to the extraction of a subsequence:

$$
\tilde{J}^{\alpha} \rightarrow J \text { in } L^{2}\left([0, T] \times \mathbb{R}^{2} \times \mathbb{R}_{+}^{2}\right)^{2} \text { weak }
$$

and therefore $J^{\alpha} \rightarrow J$ in the distributional sense. More precisely

$$
\begin{gathered}
\int_{0}^{T} \int_{\Omega^{\alpha}} \int_{\mathbb{R}_{+}^{2}} J_{1}^{\alpha} \phi d \varepsilon_{1} d \varepsilon_{2} d x d t \rightarrow \int_{0}^{T} \int_{\Omega^{\alpha}} \int_{\mathbb{R}_{+}^{2}} J_{1} \phi d \varepsilon_{1} d \varepsilon_{2} d x d t, \\
\int_{0}^{T} \int_{\Omega^{\alpha}} \int_{\mathbb{R}_{+}^{2}} J_{2}^{\alpha} \phi d \varepsilon_{1} d \varepsilon_{2} d x d t \rightarrow \int_{0}^{T} \int_{\Omega^{\alpha}} \int_{\mathbb{R}_{+}^{2}} J_{2} \phi d \varepsilon_{1} d \varepsilon_{2} d x d t,
\end{gathered}
$$

as $\alpha \rightarrow 0$ for all test functions $\phi \in C_{c}^{1}\left([0, T] \times \mathbb{R}^{2} \times \mathbb{R}_{+}^{2}\right)$.

We note that the convergence (5.22) and (5.23) allows us to pass to the limit in (5.14) and we obtain

Corollary 5.1. For any test function $\phi \in C_{c}^{2}\left([0, T] \times \mathbb{R}^{2} \times \mathbb{R}_{+}^{2}\right)$ such that $\phi(T, \cdot, \cdot, \cdot)=$ 0 , we have

$$
\begin{aligned}
& \int_{0}^{T} \int_{\mathbb{R}^{2} \times \mathbb{R}_{+}^{2}} \frac{2}{\sqrt{\varepsilon_{1} \varepsilon_{2}}} F\left(t, x, \varepsilon_{1}, \varepsilon_{2}\right) \partial_{t} \phi\left(t, x, \varepsilon_{1}, \varepsilon_{2}\right) d x d \varepsilon_{1} d \varepsilon_{2} d t \\
& +\int_{0}^{T} \int_{\mathbb{R}^{2} \times \mathbb{R}_{+}^{2}} J_{1}\left(t, x, \varepsilon_{1}, \varepsilon_{2}\right)\left(\partial_{x_{1}}+\partial_{x_{1}} V \partial_{\varepsilon_{1}}\right) \phi\left(t, x, \varepsilon_{1}, \varepsilon_{2}\right) d x d \varepsilon_{1} d \varepsilon_{2} d t \\
& \left.+\int_{0}^{T} \int_{\mathbb{R}^{2} \times \mathbb{R}_{+}^{2}} J_{2}\left(t, x, \varepsilon_{1}, \varepsilon_{2}\right)\left(\partial_{x_{2}}+\partial_{x_{2}} V \partial_{\varepsilon_{2}}\right) \phi\left(t, x, \varepsilon_{1}, \varepsilon_{2}\right)\right) d x d \varepsilon_{1} d \varepsilon_{2} d t \\
& +\int_{\mathbb{R}^{2} \times \mathbb{R}_{+}^{2}} \frac{2}{\sqrt{\varepsilon_{1} \varepsilon_{2}}} F_{I}\left(x, \varepsilon_{1}, \varepsilon_{2}\right) \phi\left(0, x, \varepsilon_{1}, \varepsilon_{2}\right) d x d \varepsilon_{1} d \varepsilon_{2}=0 .
\end{aligned}
$$

Proof of Lemma 5.5: We shall only give the detail of the proof of (5.19). We first define the current carried by the outgoing and incoming traces:

$$
\begin{aligned}
J_{V_{n}}^{\alpha, \text { out }}\left(t, x_{2}, \varepsilon_{1}, \varepsilon_{2}\right)=\frac{1}{\alpha \sqrt{2 \varepsilon_{2}}} \sum_{s_{2}=\mp 1} & {\left[\gamma_{V_{n}}^{\text {out }}\left(f^{\alpha}\right)\left(t, x_{2}, \sqrt{2 \varepsilon_{1}}, s_{2} \sqrt{2 \varepsilon_{2}}\right)\right.} \\
& \left.-\gamma_{V_{n}}^{\text {out }}\left(f^{\alpha}\right)\left(t, x_{2},-\sqrt{2 \varepsilon_{1}}, s_{2} \sqrt{2 \varepsilon_{2}}\right)\right], \\
J_{V_{n}}^{\alpha, \text { inc }}\left(t, x_{2}, \varepsilon_{1}, \varepsilon_{2}\right)=\frac{1}{\alpha \sqrt{2 \varepsilon_{2}}} \sum_{s_{2}=\mp 1} & {\left[\gamma_{V_{n}}^{\text {inc }}\left(f^{\alpha}\right)\left(t, x_{2}, \sqrt{2 \varepsilon_{1}}, s_{2} \sqrt{2 \varepsilon_{2}}\right)\right.} \\
& \left.-\gamma_{V_{n}}^{\text {inc }}\left(f^{\alpha}\right)\left(t, x_{2},-\sqrt{2 \varepsilon_{1}}, s_{2} \sqrt{2 \varepsilon_{2}}\right)\right] .
\end{aligned}
$$

Then, we construct the piecewise constant functions $J_{V}^{\alpha, o u t}, J_{V}^{\alpha, i n c}$ as follows:

$$
J_{V}^{\alpha, \text { out }}\left(t, x_{1}, x_{2}, \varepsilon_{1}, \varepsilon_{2}\right)=J_{V_{n}}^{\alpha, \text { out }}\left(t, x_{2}, \varepsilon_{1}, \varepsilon_{2}\right) ; \quad x_{1} \in\left(\left(n-\frac{1}{2}\right) \alpha,\left(n+\frac{1}{2}\right) \alpha\right), x_{2} \in \mathbb{R},
$$




$$
J_{V}^{\alpha, i n c}\left(t, x_{1}, x_{2}, \varepsilon_{1}, \varepsilon_{2}\right)=J_{V_{n}}^{\alpha, i n c}\left(t, x_{2}, \varepsilon_{1}, \varepsilon_{2}\right) ; \quad x_{1} \in\left(\left(n-\frac{1}{2}\right) \alpha,\left(n+\frac{1}{2}\right) \alpha\right), x_{2} \in \mathbb{R} .
$$

Since

$$
\gamma_{V_{n}}^{\text {out }}\left(f^{\alpha}\right)+\gamma_{V_{n}}^{i n c}\left(f^{\alpha}\right)=\gamma_{V_{n}}^{+}\left(f^{\alpha}\right)+\gamma_{V_{n}}^{-}\left(f^{\alpha}\right)
$$

it is readily seen that

$$
\tilde{J}_{V}^{\alpha}=\frac{1}{2}\left(J_{V}^{\alpha, i n c}+J_{V}^{\alpha, o u t}\right)
$$

By $(5.27)$, in order to prove that $\tilde{J}_{V}^{\alpha}$ bounded in $L^{2}\left([0, T] \times \mathbb{R}^{2}, L_{l o c}^{2}\left(\mathbb{R}_{+}^{2}\right)\right)^{2}$, it is enough to show that $J_{V}^{\alpha, o u t}, J_{V}^{\alpha, i n c}$ are separately bounded in this space. The proof follows the same lines as that of Lemma 4.5 of [4]. We shall skip it here for simplicity. In order to prove that $J_{1}^{\alpha}-\tilde{J}_{V}^{\alpha}$ in $\mathcal{D}^{\prime}$, we write

$$
\begin{aligned}
& \left\langle J_{1}^{\alpha}-\tilde{J}_{V}^{\alpha}, \varphi\right\rangle_{\mathcal{D}^{\prime}, \mathcal{D}}=\int_{0}^{T} \int_{\Omega^{\alpha}} \int_{\mathbb{R}_{+}^{2}} J_{1}^{\alpha} \varphi d x d \varepsilon_{1} d \varepsilon_{2} d t-\int_{0}^{T} \int_{\Omega^{\alpha}} \int_{\mathbb{R}_{+}} \tilde{J}_{V}^{\alpha} \varphi d x d \varepsilon_{1}, \varepsilon_{2} d t=M^{\alpha} \\
& +\int_{0}^{T} \int_{\mathbb{R}_{+}^{2}} \sum_{n \in \mathbb{Z}} \int_{\mathbb{R}} \int_{\left(n+\frac{1}{2}\right) \alpha}^{(n+1) \alpha} \gamma_{V_{n+1}}^{-}\left(J_{1}^{\alpha}\right)\left(t, x_{2}, \varepsilon_{1}, \varepsilon_{2}\right)\left[\varphi\left(t,(n+1) \alpha, x_{2}, \varepsilon_{1}, \varepsilon_{2}\right)\right. \\
& +\int_{0}^{T} \int_{\mathbb{R}_{+}^{2}} \sum_{n \in \mathbb{Z}} \int_{\mathbb{R}} \int_{n \alpha}^{\left(n+\frac{1}{2}\right) \alpha} \gamma_{V_{n}}^{+}\left(J_{1}^{\alpha}\right)\left(t, x_{2}, \varepsilon_{1}, \varepsilon_{2}\right)\left[\varphi\left(t, n \alpha, x_{2}, \varepsilon_{1}, \varepsilon_{2}\right)-\varphi\left(t, x_{1}, x_{2}, \varepsilon_{1}, \varepsilon_{2}\right)\right] \\
& -\int_{0}^{T} \int_{\mathbb{R}_{+}^{2}} \sum_{m \in \mathbb{Z}} \int_{\mathbb{R}}\left\{x_{1} d x_{2} d \varepsilon_{1} d \varepsilon_{2} d t\right. \\
& \left\{\gamma_{H_{m+1}}^{-}\left(J_{2}^{\alpha}\right)\left(t, x_{1}, \varepsilon_{1}, \varepsilon_{2}\right) \varphi\left(t, x_{1},(m+1) \alpha, \varepsilon_{1}, \varepsilon_{2}\right)\right. \\
& \left.-\gamma_{H_{m}}^{+}\left(J_{2}^{\alpha}\right)\left(t, x_{1}, \varepsilon_{1}, \varepsilon_{2}\right) \varphi\left(t, x_{1}, m \alpha, \varepsilon_{1}, \varepsilon_{2}\right)\right\} \psi^{\alpha}\left(x_{1}\right) d x_{1} d \varepsilon_{1} d \varepsilon_{2} d t .
\end{aligned}
$$

The last term of the right hand side of this identity can be estimated as follows

$$
\begin{aligned}
& \int_{0}^{T} \int_{K} \sum_{m \in \mathbb{Z}} \int_{\mathbb{R}}\left|\gamma_{H_{m+1}}^{\mp}\left(J_{2}^{\alpha}\right)\left(t, x_{1}, \varepsilon_{1}, \varepsilon_{2}\right) \varphi\left(t, x_{1},(m+1) \alpha, \varepsilon_{1}, \varepsilon_{2}\right) \psi^{\alpha}\left(x_{1}\right)\right| d x_{1} d \varepsilon_{1} d \varepsilon_{2} d t \\
& \leq \alpha^{1 / 2} N(\varphi)\left[\left\|J_{H}^{\alpha, \text { out }}\right\|_{L^{2}\left([0, T] \times \mathbb{R}^{2}, L_{L o c}^{2}\left(\mathbb{R}_{+}^{2}\right)\right)}^{2}+\left\|J_{H}^{\alpha, \text { inc }}\right\|_{L^{2}\left([0, T] \times \mathbb{R}^{2}, L_{L o c}^{2}\left(\mathbb{R}_{+}^{2}\right)\right)}^{2}\right]^{\frac{1}{2}},
\end{aligned}
$$

The other terms can be treated exactly as in the one-dimensional case (up to an additional integration with respect to $x_{2}, v_{2}$ ) and are shown to converge to zero as $\alpha$ tends to zero. This finally proves that $\lim _{\alpha \rightarrow 0}\left(J_{1}^{\alpha}-\tilde{J}_{V}^{\alpha}\right)=0$ in $\mathcal{D}^{\prime}$

The aim of the next subsection is to derive Equation (2.11) for the current.

5.4. Equation for the current. We first prove that there exists $\chi_{V}(x, v)$ and $\chi_{H}(x, v)$ such that:

$$
\begin{aligned}
& \left\{\begin{array}{l}
\left(I-\mathcal{B}^{V}\right) \chi_{V}=\frac{1}{2}\left(I+\mathcal{B}^{V}\right) \operatorname{sgn}\left(v_{1}\right), \\
\left(I-\mathcal{B}^{H}\right) \chi_{V}=0
\end{array}\right. \\
& \left\{\begin{array}{l}
\left(I-\mathcal{B}^{H}\right) \chi_{H}=\frac{1}{2}\left(I+\mathcal{B}^{H}\right) \operatorname{sgn}\left(v_{2}\right), \\
\left(I-\mathcal{B}^{V}\right) \chi_{H}=0 .
\end{array}\right.
\end{aligned}
$$


Lemma 5.6. Problem (5.28) (respectively (5.29)) has a unique solution $\chi_{V}$ (respectively $\chi_{H}$ ) in the space of odd functions with respect to $v_{1}$ (respectively $v_{2}$ ) and even with respect to $v_{2}$ (respectively $\left.v_{1}\right)$. Both $\chi_{V}, \chi_{H}$ are in $C^{1}\left(\mathbb{R}^{2} \times \mathbb{R}^{2}\right)$.

Proof: Since $\mathcal{B}^{V}$ has the simple form given in (2.4) and (2.5), we deduce from Hypothesis 2.3 that the unique solution of (5.28) which is odd with respect to $v_{1}$ is

$$
\chi_{V}(x, v)=\frac{\mathcal{T}^{V}\left(x, \varepsilon_{1}, \varepsilon_{2}\right)}{2\left(1-\mathcal{T}^{V}\left(x, \varepsilon_{1}, \varepsilon_{2}\right)\right)} \operatorname{sgn}\left(v_{1}\right) .
$$

It is readily seen that $\chi_{V}$ is even with respect to $v_{2}$ which implies

$$
\left(I-\mathcal{B}^{H}\right) \chi_{V}=0 .
$$

In the same way, we have

$$
\chi_{H}(x, v)=\frac{\mathcal{T}^{H}\left(x, \varepsilon_{1}, \varepsilon_{2}\right)}{2\left(I-\mathcal{T}^{H}\left(x, \varepsilon_{1}, \varepsilon_{2}\right)\right)} \operatorname{sgn}\left(v_{2}\right) .
$$

From the regularity of $\mathcal{T}^{V}$ (respectively $\mathcal{T}^{H}$ ) (Hypothesis 2.3), we obtain the regularity of $\chi_{V}$ (respectively $\chi_{H}$ ).

Let us now establish the equation for the current.

LEMma 5.7. The functions $F$ and $J$ satisfy the current equation (2.11) in the distributional sense.

Proof: In order to derive the current equation (2.11), we use the weak formulation (5.10) with $\left(\chi_{V}(x, v)+\chi_{H}(x, v)\right) \psi\left(t, x, \varepsilon_{1}, \varepsilon_{2}\right)$ as a test function and obtain

$$
\begin{aligned}
& \left.\frac{1}{\alpha} \int_{0}^{T} \sum_{n \in \mathbb{Z}} \alpha \int_{\mathbb{R}} \int_{\mathbb{R}^{2}} \gamma_{V_{n}}^{\text {out }}\left(f^{\alpha}\right)\left(t, x_{2}, v\right) \psi\left(t, n \alpha, x_{2}, \varepsilon_{1}, \varepsilon_{2}\right)\right) \\
& \left.+\frac{1}{\alpha} \int_{0}^{T} \sum_{m \in \mathbb{Z}} \alpha \int_{\mathbb{R}} \int_{\mathbb{R}^{2}} \gamma_{H_{m}}^{\text {out }}\left(f^{\alpha}\right)\left(t, x_{1}, v\right) \psi\left(t, x_{1}, m \alpha, \varepsilon_{1}, \varepsilon_{2}\right)\right) \\
& \left.=\alpha \int_{0}^{T} \int_{\mathcal{O}^{\alpha}} f^{\alpha}(t, x, v) \partial_{t}\left[\left(\chi_{V}(x, v)+\chi_{H}(x, v)\right) \psi\left(t, x, \varepsilon_{1}, \varepsilon_{2}\right)\right)\right] d x d v d t \\
& +\alpha \int_{\mathcal{O}^{\alpha}}^{f_{I}^{\alpha}(x, v)\left(\chi_{V}(x, v)+\chi_{H}(x, v)\right) \psi\left(0, x, \varepsilon_{1}, \varepsilon_{2}\right) d x d v} \\
& +\int_{0}^{T} \int_{\mathcal{O}^{\alpha}} f^{\alpha}(t, x, v)\left[v \cdot \nabla_{x}+\nabla_{x} V \cdot \nabla_{v}\right]\left[\left(\chi_{V}(x, v)+\chi_{H}(x, v)\right) \psi\left(t, x, \varepsilon_{1}, \varepsilon_{2}\right)\right] d x d v d t .
\end{aligned}
$$

Let us first consider the right-hand side of (5.30). The first two terms are multiplied by $\alpha$ and obviously tend to zero. Since both $F=\lim f^{\alpha}$ and $\psi$ only depend on $\varepsilon_{1}, \varepsilon_{2}$, we claim that the third term has the following limit

$$
\begin{aligned}
\lim _{\alpha \rightarrow 0} & \int_{0}^{T} \int_{\mathcal{O}^{\alpha}} f^{\alpha}(t, x, v)\left(v \cdot \nabla_{x}+\nabla_{x} V \cdot \nabla_{v}\right)\left[\left(\chi_{V}(x, v)+\chi_{H}(x, v)\right) \psi\left(t, x, \varepsilon_{1}, \varepsilon_{2}\right)\right] d v d x d t \\
& =\int_{0}^{T} \int_{\mathbb{R}^{2}} \int_{\mathbb{R}_{+}^{2}} F\left(t, x, \varepsilon_{1}, \varepsilon_{2}\right)\left\{\left(\partial_{x_{1}}+\partial_{x_{1}} V \partial_{\varepsilon_{1}}\right)\left[\psi\left(t, x, \varepsilon_{1}, \varepsilon_{2}\right) D_{11}\left(x, \varepsilon_{1}, \varepsilon_{2}\right)\right]\right. \\
& \left.+\left(\partial_{x_{2}}+\partial_{x_{2}} V \partial_{\varepsilon_{2}}\right)\left[\psi\left(t, x, \varepsilon_{1}, \varepsilon_{2}\right) D_{22}\left(x, \varepsilon_{1}, \varepsilon_{2}\right)\right]\right\} d x d \varepsilon_{1} d \varepsilon_{2} d t,
\end{aligned}
$$


where

$$
\begin{aligned}
& D_{11}\left(x, \varepsilon_{1}, \varepsilon_{2}\right)=\frac{4}{\sqrt{2 \varepsilon_{2}}} \chi_{V}\left(x, \sqrt{2 \varepsilon_{1}}, \sqrt{2 \varepsilon_{2}}\right), \\
& D_{22}\left(x, \varepsilon_{1}, \varepsilon_{2}\right)=\frac{4}{\sqrt{2 \varepsilon_{1}}} \chi_{H}\left(x, \sqrt{2 \varepsilon_{1}}, \sqrt{2 \varepsilon_{2}}\right),
\end{aligned}
$$

Indeed, using the oddness- evenness properties of $\chi_{V}, \chi_{H}$ with respect to $v_{1}$ and $v_{2}$, we have

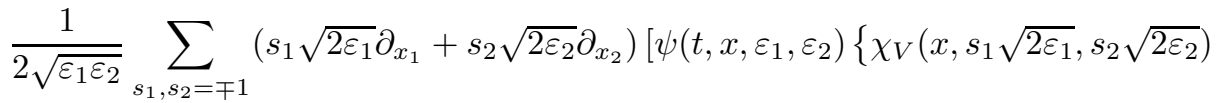

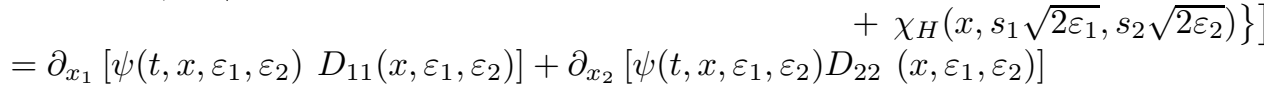

and

$$
\begin{aligned}
& \frac{1}{2 \sqrt{\varepsilon_{1} \varepsilon_{2}}} \sum_{s_{1}, s_{2}=\mp 1} \nabla_{x} V \cdot \nabla_{v}\left[\left(\chi_{V}\left(x, s_{1} \sqrt{2 \varepsilon_{1}}, s_{2} \sqrt{2 \varepsilon_{2}}\right)\right.\right. \\
& \left.\quad+\chi_{H}\left(x, s_{1} \sqrt{2 \varepsilon_{1}}, s_{2} \sqrt{2 \varepsilon_{2}}\right) \psi\left(t, x, \varepsilon_{1}, \varepsilon_{2}\right)\right] \\
& =\partial_{x_{1}} V \partial_{\varepsilon_{1}}\left[\psi\left(t, x, \varepsilon_{1}, \varepsilon_{2}\right) D_{11}\left(x, \varepsilon_{1}, \varepsilon_{2}\right)\right]+\partial_{x_{2}} V \partial_{\varepsilon_{2}}\left[\psi\left(t, x, \varepsilon_{1}, \varepsilon_{2}\right) D_{22}\left(x, \varepsilon_{1}, \varepsilon_{2}\right)\right] .
\end{aligned}
$$

Let us now deal with the left-hand side of (5.30). It is readily seen that

$$
\begin{aligned}
& \frac{1}{\alpha} \int_{0}^{T} \sum_{m \in \mathbb{Z}} \alpha \int_{\mathbb{R}} \int_{\mathbb{R}^{2}} \begin{array}{l}
\left.\gamma_{H_{m}}^{\text {out }}\left(f^{\alpha}\right)\left(t, x_{1}, v\right) \psi\left(t, n \alpha, x_{2}, \varepsilon_{1}, \varepsilon_{2}\right)\right) \\
\left(I-\mathcal{B}^{V}\left(n \alpha, x_{2}\right)\right)\left(\chi_{V}\left(n \alpha, x_{2}, v\right)+\chi_{H}\left(n \alpha, x_{2} v\right)\right)\left|v_{1}\right| d v d x_{1} d t \\
=\int_{0}^{T} \sum_{n \in \mathbb{Z}} \alpha \int_{\mathbb{R}} \int_{\mathbb{R}^{2}} \frac{1}{\alpha} P_{V} \gamma_{V_{n}}^{\text {out }}\left(f^{\alpha}\right)\left(t, x_{2}, v\right)\left(I-\mathcal{B}^{V}\left(n \alpha, x_{2}\right)\right) \chi_{V}\left(n \alpha, x_{2}, v\right) \\
\left.\psi\left(t, n \alpha, x_{2}, \varepsilon_{1}, \varepsilon_{2}\right)\right)\left|v_{1}\right| d v d x_{2} d t
\end{array}
\end{aligned}
$$

and

$$
\begin{aligned}
& \left.\frac{1}{\alpha} \int_{0}^{T} \sum_{m \in \mathbb{Z}} \alpha \int_{\mathbb{R}} \int_{\mathbb{R}^{2}} \gamma_{H_{m}}^{\text {out }}\left(f^{\alpha}\right)\left(t, x_{1}, v\right) \psi\left(t, x_{1}, m \alpha, \varepsilon_{1}, \varepsilon_{2}\right)\right) \\
& \left(I-\mathcal{B}^{H}\left(x_{1}, m \alpha\right)\right)\left(\chi_{V}\left(x_{1}, m \alpha, v\right)+\chi_{H}\left(x_{1}, m \alpha, v\right)\right)\left|v_{2}\right| d v d x_{1} d t \\
& =\int_{0}^{T} \sum_{n \in \mathbb{Z}} \alpha \int_{\mathbb{R}} \int_{\mathbb{R}^{2}} \frac{1}{\alpha} P_{H} \gamma_{H_{m}}^{\text {out }}\left(f^{\alpha}\right)\left(t, x_{1}, v\right)\left(I-\mathcal{B}^{H}\left(x_{1}, m \alpha\right)\right) \chi_{H}\left(x_{1}, m \alpha, v\right) \\
& \left.\psi\left(t, x_{1}, m \alpha, \varepsilon_{1}, \varepsilon_{2}\right)\right)\left|v_{2}\right| d v d x_{2} d t .
\end{aligned}
$$

Defining the piecewise constant functions $g_{V}^{\alpha, o u t}, g_{V}^{\alpha, i n c}, g_{H}^{\alpha, \text { out }}$ and $g_{H}^{\alpha, i n c}$ by

$$
\begin{array}{ll}
g_{V}^{\alpha, \text { out }}\left(t, x_{1}, x_{2}, v\right)=\frac{1}{\alpha} P_{V} \gamma_{V_{n}}^{\text {out }}\left(f^{\alpha}\right)\left(t, x_{2}, v\right) ; & x_{1} \in\left(\left(n-\frac{1}{2}\right) \alpha,\left(n+\frac{1}{2}\right) \alpha\right), x_{2} \in \mathbb{R}, \\
g_{V}^{\alpha, \text { inc }}\left(t, x_{1}, x_{2}, v\right)=\frac{1}{\alpha} P_{V} \gamma_{V_{n}}^{\text {inc }}\left(f^{\alpha}\right)\left(t, x_{2}, v\right) ; & x_{1} \in\left(\left(n-\frac{1}{2}\right) \alpha,\left(n+\frac{1}{2}\right) \alpha\right), x_{2} \in \mathbb{R}, \\
g_{H}^{\alpha, \text { out }}\left(t, x_{1}, x_{2}, v\right)=\frac{1}{\alpha} P_{H} \gamma_{H_{m}}^{\text {out }}\left(f^{\alpha}\right)\left(t, x_{1}, v\right) ; & x_{1} \in \mathbb{R}, x_{2} \in\left(\left(m-\frac{1}{2}\right) \alpha,\left(m+\frac{1}{2}\right) \alpha\right),
\end{array}
$$


$g_{H}^{\alpha, i n c}\left(t, x_{1}, x_{2}, v\right)=\frac{1}{\alpha} P_{H} \gamma_{H_{m}}^{i n c}\left(f^{\alpha}\right)\left(t, x_{1}, v\right) ; \quad x_{1} \in \mathbb{R}, x_{2} \in\left(\left(m-\frac{1}{2}\right) \alpha,\left(m+\frac{1}{2}\right) \alpha\right)$,

we deduce from estimates (5.2) and (5.3), that $\sqrt{\left|v_{1}\right|} g_{V}^{\alpha, \text { out }}, \sqrt{\left|v_{1}\right|} g_{V}^{\alpha, \text { inc }}, \sqrt{\left|v_{2}\right|} g_{H}^{\alpha, \text { out }}$ and $\sqrt{\left|v_{2}\right|} g_{H}^{\alpha, \text { out }}$ are bounded in $L^{2}\left([0, T] \times \mathbb{R}^{2} \times \mathbb{R}^{2}\right)$. Let $\sqrt{\left|v_{1}\right|} g_{V}^{0, \text { out }}, \sqrt{\left|v_{1}\right|} g_{V}^{0, \text { inc }}$, $\sqrt{\left|v_{2}\right|} g_{H}^{0, \text { out }}$ and $\sqrt{\left|v_{2}\right|} g_{H}^{0, \text { out }}$ be their weak limits (up to the extraction of a subsequence). We deduce from the identities

$$
\begin{gathered}
P_{V} \gamma_{V_{n}}^{i n c}\left(f^{\alpha}\right)=\mathcal{B}^{V}\left(n \alpha, x_{2}\right) P_{V} \gamma_{V_{n}}^{\text {out }}\left(f^{\alpha}\right), \\
P_{H} \gamma_{H_{m}}^{\text {inc }}\left(f^{\alpha}\right)=\mathcal{B}^{H}\left(x_{1}, m \alpha\right) P_{H} \gamma_{H_{m}}^{\text {out }}\left(f^{\alpha}\right),
\end{gathered}
$$

that

$$
g_{V}^{0, \text { inc }}=\mathcal{B}^{V}(x) g_{V}^{0, \text { out }} \quad, \quad g_{H}^{0, \text { inc }}=\mathcal{B}^{H}(x) g_{V}^{0, \text { out }}
$$

In (5.32) and (5.33), the factors $\left(I-\mathcal{B}^{V}\left(n \alpha, x_{2}, v\right)\right)\left(\chi_{V}\left(n \alpha, x_{2}, v\right)+\chi_{H}\left(n \alpha, x_{2}, v\right)\right) \psi(t$, $\left.\left.n \alpha, x_{2}, \varepsilon_{1}, \varepsilon_{2}\right)\right)$ (respectively $\left(I-\mathcal{B}^{H}\left(x_{1}, m \alpha, v\right)\right)\left(\chi_{V}\left(x_{1}, m \alpha, v\right)+\chi_{H}\left(x_{1}, m \alpha, v\right)\right) \psi(t$, $\left.\left.x_{1}, m \alpha, \varepsilon_{1}, \varepsilon_{2}\right)\right)$ )are the piecewise constant approximation of the functions $\left(I-\mathcal{B}^{V}(x\right.$, $\left.v))\left(\chi_{V}(x, v)+\chi_{H}(x, v)\right) \psi\left(t, x, \varepsilon_{1}, \varepsilon_{2}\right)\right)$ (respectively $\left(I-\mathcal{B}^{H}(x, v)\right)\left(\chi_{V}(x, v)+\chi_{H}(x\right.$, $\left.\left.v)) \psi\left(t, x, \varepsilon_{1}, \varepsilon_{2}\right)\right)\right)$. Since these functions are uniformly continuous with respect to $x$, with values in $L^{2}\left(\mathbb{R}^{2}\right)$, the piecewise constant approximation is an approximation in the strong topology of $L^{2}\left([0, T] \times \mathbb{R}^{2}, L^{2}\left(\mathbb{R}^{2}\right)\right)$. Therefore, the expressions (5.32) (respectively (5.33)) are the $L^{2}$ product of a weakly converging sequence with a strongly converging one. Therefore, they converge as $\alpha \rightarrow 0$ respectively to:

$$
\left.\int_{0}^{T} \int_{\mathbb{R}^{2}} \int_{\mathbb{R}^{2}} g_{V}^{0, \text { out }}(t, x, v)\left(I-\mathcal{B}^{V}(x, v)\right) \chi_{V}(x, v) \psi\left(t, x, \varepsilon_{1}, \varepsilon_{2}\right)\right)\left|v_{1}\right| d v d x d t
$$

and

$$
\left.\int_{0}^{T} \int_{\mathbb{R}^{2}} \int_{\mathbb{R}^{2}} g_{H}^{0, \text { out }}(t, x, v)\left(I-\mathcal{B}^{H}(x, v)\right) \chi_{H}(x, v) \psi\left(t, x, \varepsilon_{1}, \varepsilon_{2}\right)\right)\left|v_{2}\right| d v d x d t .
$$

Introducing

$$
\begin{aligned}
& J_{V}\left(t, x, \varepsilon_{1}, \varepsilon_{2}\right)=\frac{1}{\sqrt{2 \varepsilon_{2}}} \sum_{s_{1}, s_{2}=\mp 1}\left[\left(g_{V}^{0, \text { out }}\left(I-\mathcal{B}^{V}\right) \chi_{V}\right)\left(x, s_{1} \sqrt{2 \varepsilon_{1}}, s_{2} \sqrt{2 \varepsilon_{2}}\right)\right], \\
& J_{H}\left(t, x, \varepsilon_{1}, \varepsilon_{2}\right)=\frac{1}{\sqrt{2 \varepsilon_{1}}} \sum_{s_{1}, s_{2}=\mp 1}\left[\left(g_{H}^{0, \text { out }}\left(I-\mathcal{B}^{H}\right) \chi_{H}\right)\left(t x, s_{1} \sqrt{2 \varepsilon_{1}}, s_{2} \sqrt{2 \varepsilon_{2}}\right)\right],
\end{aligned}
$$

(5.34), (5.35) and (5.31) yield

$$
\begin{array}{ll}
\int_{0}^{T} \int_{\mathbb{R}^{2}} \int_{\mathbb{R}_{+}^{2}} & \left(J_{V}+J_{H}\right) \psi\left(t, x, \varepsilon_{1}, \varepsilon_{2}\right) d x d \varepsilon_{1} d \varepsilon_{2} d t \\
\left.=\int_{0}^{T} \int_{\mathbb{R}^{2}} \int_{\mathbb{R}_{+}^{2}} F\left(t, x, \varepsilon_{1}, \varepsilon_{2}\right)\right)\left\{\left(\partial_{x_{1}}+\partial_{x_{1}} V \partial_{\varepsilon_{1}}\right)\left[\psi\left(t, x, \varepsilon_{1}, \varepsilon_{2}\right) D_{11}\right]\right. \\
& \left.+\left(\partial_{x_{2}}+\partial_{x_{2}} V \partial_{\varepsilon_{2}}\right)\left[\psi\left(t, x, \varepsilon_{1}, \varepsilon_{2}\right) D_{22}\right]\right\} d x d \varepsilon d t
\end{array}
$$


which implies that

$$
\begin{aligned}
& J_{V}=D_{11}\left(\partial_{x_{1}}+\partial_{x_{1}} V \partial_{\varepsilon_{1}}\right) F, \\
& J_{H}=D_{22}\left(\partial_{x_{2}}+\partial_{x_{2}} V \partial_{\varepsilon_{2}}\right) F
\end{aligned}
$$

in $D^{\prime}\left((0, T) \times \mathbb{R}^{2} \times \mathbb{R}_{+}^{2}\right)$. Using the definition of $\chi_{V}$, we find

$$
\begin{aligned}
& J_{V}\left(t, x, \varepsilon_{1}, \varepsilon_{2}\right)=\frac{1}{\sqrt{2 \varepsilon_{2}}} \sum_{s_{1}, s_{2}=\mp 1}\left[\left(g_{V}^{0, \text { out }}\left(I-\mathcal{B}^{V}\right) \chi_{V}\right)\left(t, x, s_{1} \sqrt{2 \varepsilon_{1}}, s_{2} \sqrt{2 \varepsilon_{2}}\right)\right] \\
& =\frac{1}{\sqrt{2 \varepsilon_{2}}} \sum_{s_{2}=\mp 1}\left[\frac{1}{2}\left(I+\mathcal{B}^{V}\right) g_{V}^{0, \text { out }}\left(t, x, \sqrt{2 \varepsilon_{1}}, s_{2} \sqrt{2 \varepsilon_{2}}\right)\right. \\
& \left.\quad-\frac{1}{2}\left(I+\mathcal{B}^{V}\right) g_{V}^{0, \text { out }}\left(t, x,-\sqrt{2 \varepsilon_{1}}, s_{2} \sqrt{2 \varepsilon_{2}}\right)\right] \\
& =\frac{1}{2 \sqrt{2 \varepsilon_{2}}} \sum_{s_{2}=\mp 1}\left[\left(g_{V}^{0, \text { out }}+g_{V}^{0, \text { inc }}\right)\left(t, x, \sqrt{2 \varepsilon_{1}}, s_{2} \sqrt{2 \varepsilon_{2}}\right)\right. \\
& \left.\quad-\left(g_{V}^{0, \text { out }}+g_{V}^{0, \text { inc }}\right)\left(t, x,-\sqrt{2 \varepsilon_{1}}, s_{2} \sqrt{2 \varepsilon_{2}}\right)\right] .
\end{aligned}
$$

Besides, the weak convergence of $g_{V}^{\alpha, \text { out }}, g_{V}^{\alpha, \text { inc }}$ towards $g_{V}^{0, \text { out }}, g_{V}^{0, \text { inc }}$ implies that

$$
\begin{aligned}
& \frac{1}{\sqrt{2 \varepsilon_{2}}} \sum_{s_{2}=\mp 1}\left[\left(g_{V}^{0, \text { out }}+g_{V}^{0, \text { inc }}\right)\left(t, x, \sqrt{2 \varepsilon_{1}}, s_{2} \sqrt{2 \varepsilon_{2}}\right)\right. \\
& \left.\quad-\left(g_{V}^{0, \text { out }}+g_{V}^{0, \text { inc }}\right)\left(t, x,-\sqrt{2 \varepsilon_{1}}, s_{2} \sqrt{2 \varepsilon_{2}}\right)\right]= \\
& \text { weak } \lim _{\alpha \rightarrow 0} \frac{1}{\sqrt{2 \varepsilon_{2}}} \sum_{s_{2}=\mp 1}\left[\left(g_{V}^{\alpha, \text { out }}+g_{V}^{\alpha, \text { inc }}\right)\left(t, x, \sqrt{2 \varepsilon_{1}}, s_{2} \sqrt{2 \varepsilon_{2}}\right)\right. \\
& \left.\quad-\left(g_{V}^{\alpha, \text { out }}+g_{V}^{\alpha, \text { inc }}\right)\left(t, x,-\sqrt{2 \varepsilon_{1}}, s_{2} \sqrt{2 \varepsilon_{2}}\right)\right] .
\end{aligned}
$$

Moreover, we deduce from (5.27) that

$$
\begin{aligned}
& \frac{1}{2 \sqrt{2 \varepsilon_{2}}} \sum_{s_{2}=\mp 1}\left[\left(g_{V}^{\alpha, \text { out }}+g_{V}^{\alpha, \text { inc }}\right)\left(t, x, \sqrt{2 \varepsilon_{1}}, s_{2} \sqrt{2 \varepsilon_{2}}\right)\right. \\
& \left.\quad-\left(g_{V}^{\alpha, \text { out }}+g_{V}^{\alpha, \text { inc }}\right)\left(t, x,-\sqrt{2 \varepsilon_{1}}, s_{2} \sqrt{2 \varepsilon_{2}}\right)\right] \\
& =\tilde{J}_{V}^{\alpha}\left(t, x, \varepsilon_{1}, \varepsilon_{2}\right) .
\end{aligned}
$$

which proves that

$$
J_{V}\left(t, x, \varepsilon_{1}, \varepsilon_{2}\right)=\text { weak } \lim _{\alpha \rightarrow 0} \tilde{J}_{V}^{\alpha}\left(t, x, \varepsilon_{1}, \varepsilon_{2}\right) .
$$

A similar argument leads to

$$
J_{H}\left(t, x, \varepsilon_{1}, \varepsilon_{2}\right)=\text { weak } \lim _{\alpha \rightarrow 0} \tilde{J}_{H}^{\alpha}\left(t, x, \varepsilon_{1}, \varepsilon_{2}\right) .
$$

We finally deduce from (5.21) that

$$
J_{V}=J_{1} ; \quad J_{H}=J_{2} .
$$




\section{Extension to rough interfaces}

The interfaces between two grains have been assumed to be perfectly linear and perfectly clean, in such a way that the reflection obeys Descartes law. In practice, this is not the case and therefore the reflection may be diffusive. A particle hitting the grain boundary can be scattered randomly. In this section, we treat the diffusive part as a perturbation of the full scattering mechanism. More precisely the collision operator at the interface writes

$$
\mathcal{B}^{\alpha}=(1-\alpha) \mathcal{B}+\alpha \tilde{\mathcal{B}}
$$

where

$$
\tilde{\mathcal{B}} \varphi(x, v)=\int_{\mathbb{R}^{2}} \sigma\left(v, v^{\prime}\right) \varphi\left(x, v^{\prime}\right) \delta\left(|v|^{2}-\left|v^{\prime}\right|^{2}\right) d v^{\prime}
$$

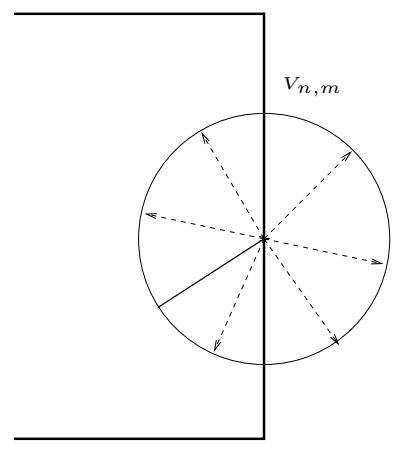

FIG. 6.1. Diffusive reflection-transmission at the grain boundary

The diffusive reflection is assumed to be elastic ( the electrons do not lose energy when they hit the grain boundary). The perturbed problem is

$$
\left\{\begin{array}{l}
\alpha \partial_{t} f^{\alpha}+v \cdot \nabla_{x} f^{\alpha}+\nabla_{x} V \cdot \nabla_{v} f^{\alpha}=0 \quad x \in \Omega^{\alpha}, v \in \mathbb{R}^{2}, t \geq 0 \\
\gamma^{\text {inc }} f^{\alpha}=\mathcal{B}^{\alpha} \gamma^{\text {out }} f^{\alpha} .
\end{array}\right.
$$

We shall prove, that in the limit the distribution function relaxes towards a function of the total energy $\varepsilon=\varepsilon_{1}+\varepsilon_{2}$ whereas the diffusion coefficient is deduced from the diffusion coefficients $D_{i i}\left(x, \varepsilon_{1}, \varepsilon_{2}\right)$ derived in the previous sections. The idea is that $\alpha \tilde{\mathcal{B}}$ is strong enough to induce a change in the equilibrium state but not to influence the diffusion equation. This fact has first been noticed for the diffusion approximation of the Boltzmann equation in [10]. 


\section{HYPOTHESIS 6.1.}

There exists a smooth function $F_{I}(x, \varepsilon)$ defined on $\mathbb{R}^{2} \times \mathbb{R}_{+}$, satisfying:

$$
F_{I}(x, \varepsilon) \in L^{2}\left(\mathbb{R}^{2} \times \mathbb{R}_{+}\right), \quad\left(v \cdot \nabla_{x}+\nabla_{x} V \cdot \nabla_{v}\right) F_{I}(x, \varepsilon) \in L^{2}\left(\mathbb{R}^{2} \times \mathbb{R}_{+}\right),
$$

where $\varepsilon=\frac{1}{2}\left(v_{1}^{2}+v_{2}^{2}\right)$ and such that

$$
f_{I}^{\alpha}(x, v)=F_{I}(x, \varepsilon), \quad x \in \Omega^{\alpha}, v \in \mathbb{R}^{2} .
$$

Let us denote by $\mathcal{S}_{\varepsilon}$ the constant energy $\mathcal{S}_{\varepsilon}=\left\{v \in \mathbb{R}^{2}, \varepsilon(v)=\varepsilon\right\}$ and by $d \sigma(v)$ the corresponding surface measure induced by the Lebesgue one. For any continuous function $\psi$ defined on $\mathbb{R}^{2}$, we have the co-area formula

$$
\int_{\mathbb{R}^{2}} \psi(v) d v=\int_{\mathbb{R}_{+}} \int_{v \in \mathcal{S}_{\varepsilon}} \psi(v) \frac{d \sigma(v)}{|\nabla \varepsilon(v)|} d \varepsilon=\int_{\mathbb{R}_{+}}\left(\int_{0}^{2 \pi} \psi(\sqrt{2 \varepsilon} \cos \theta, \sqrt{2 \varepsilon} \sin \theta) d \theta\right) d \varepsilon .
$$

For $\varepsilon \in \mathbb{R}_{+}$, we define $L_{\delta, i}^{2}\left(\mathcal{S}_{\varepsilon}\right)$ as the weighted $L^{2}$ space, equipped with norm

$$
|u|_{L_{\delta, i}^{2}\left(\mathcal{S}_{\varepsilon}\right)}^{2}=\int_{0}^{2 \pi}|u(\sqrt{2 \varepsilon} \cos \theta, \sqrt{2 \varepsilon} \sin \theta)|^{2}\left|\omega_{i}^{\delta}(\sqrt{2 \varepsilon} \cos \theta, \sqrt{2 \varepsilon} \sin \theta)\right| d \theta
$$

so that $L_{\delta, i}^{2}\left(\mathbb{R}^{2}\right)=L^{2}\left(\mathbb{R}_{+}, L_{\delta, i}^{2}\left(\mathcal{S}_{\varepsilon}\right)\right)$. Similarly, we define $L_{i}^{2}\left(\mathcal{S}_{\varepsilon}\right)$, by changing $\omega_{i}^{\delta}\left(v_{1}, v_{2}\right)$ by $\left|v_{i}\right|$.

\section{HyPOTHESIS 6.2.}

i) Positivity: The cross section $\sigma$ is assumed to be positive almost everywhere.

ii) Particle conservation

$$
\int_{\mathbb{R}^{2}} \sigma\left(v, v^{\prime}\right)\left|v_{j}\right| \delta\left(|v|^{2}-\left|v^{\prime}\right|^{2}\right) d v^{\prime}=1
$$

for $j=1,2$.

iii)Reciprocity: $\sigma\left(v, v^{\prime}\right)=\sigma\left(-v,-v^{\prime}\right)$.

iv) $\tilde{\mathcal{B}}$ is a compact operator on $L_{1}^{2}\left(\mathcal{S}_{\varepsilon}\right) \times L_{2}^{2}\left(\mathcal{S}_{\varepsilon}\right)$.

We denote by $\tilde{Q}_{V}$ ( respectively $\tilde{Q}_{H}$ ) the orthogonal projector of $L_{1}^{2}\left(\mathcal{S}_{\varepsilon}\right)$ (respectively $\left.L_{2}^{2}\left(\mathcal{S}_{\varepsilon}\right)\right)$ onto the space of constant functions and introduce $\tilde{P}_{V}=I-\tilde{Q}_{V}$ (respectively $\tilde{P}_{H}=I-\tilde{Q}_{H}$ ) where $I$ is the identity on $L_{1}^{2}\left(\mathcal{S}_{\varepsilon}\right)$ (respectively $L_{2}^{2}\left(\mathcal{S}_{\varepsilon}\right)$ ). We denote by

$$
\tilde{Q}=\left\{\begin{array}{ll}
\tilde{Q}_{V}, & \text { on } V_{n}, \\
\tilde{Q}_{H}, & \text { on } H_{m},
\end{array} \quad \tilde{P}= \begin{cases}\tilde{P}_{V}, & \text { on } V_{n} \\
\tilde{P}_{H}, & \text { on } H_{m}\end{cases}\right.
$$

Lemma 6.1. The operators $\tilde{Q}, \tilde{P}, \tilde{\mathcal{B}}$ and $\mathcal{B}$ satisfy the following identities

$$
\begin{aligned}
& \tilde{P} \tilde{\mathcal{B}}=\tilde{\mathcal{B}} \tilde{P}, \quad \tilde{Q} \tilde{\mathcal{B}}=\tilde{\mathcal{B}} \tilde{Q}=\tilde{Q} \\
& \tilde{P} \mathcal{B}=\mathcal{B} \tilde{P}, \quad \tilde{Q} \mathcal{B}=\mathcal{B} \tilde{Q}=\tilde{Q} .
\end{aligned}
$$

Moreover, there exists $K(x, \varepsilon)<1$ such that

$$
\left\|\tilde{\mathcal{B}}^{V} \tilde{P}_{V}\right\|_{\mathcal{L}\left(L_{1}^{2}\left(\mathcal{S}_{\varepsilon}\right)\right)} \leq K(x, \varepsilon)<1,\left\|\mathcal{B}^{H} \tilde{P}_{H}\right\|_{\mathcal{L}\left(L_{2}^{2}\left(\mathcal{S}_{\varepsilon}\right)\right)} \leq K(x, \varepsilon)<1 .
$$


We have

$$
\|\tilde{\mathcal{B}}\|_{\mathcal{L}\left(L_{1}^{2}\left(\mathcal{S}_{\varepsilon}\right) \times L_{2}^{2}\left(\mathcal{S}_{\varepsilon}\right)\right)} \leq 1
$$

Hypothesis 6.3. There exists $K<1$ such that

$$
\left\|\tilde{\mathcal{B}}^{V} \tilde{P}_{V}\right\|_{\mathcal{L}\left(L_{1}^{2}\left(\mathcal{S}_{\varepsilon}\right)\right)} \leq K<1,\left\|\mathcal{B}^{H} \tilde{P}_{H}\right\|_{\mathcal{L}\left(L_{2}^{2}\left(\mathcal{S}_{\varepsilon}\right)\right)} \leq K<1, \quad \forall(x, \varepsilon) \in \mathbb{R}^{2} \times \mathbb{R}_{+} .
$$

THEOREM 6.1. i) Under the hypotheses listed in the current and previous sections (namely hypotheses 2.2, 2.3, 6.2, 6.1, 6.3), the problem (6.1) has a solution $f^{\alpha}$.

ii) When $\alpha$ tends to zero, $f^{\alpha}$ converges to $f^{0}$ in the weak star topology of $L^{\infty}([0, T]$, $L^{2}\left(\mathbb{R}^{2} \times \mathbb{R}_{+}\right)$) for any $T>0$. The limit $f^{0}$ takes the form $f^{0}(t, x, v)=F(t, x, \varepsilon)$ when $F(t, x, \varepsilon)$ is a weak solution of the problem (SHE model) posed on the domain $\mathbb{R}^{2} \times \mathbb{R}_{+}$:

$$
\left\{\begin{array}{l}
2 \pi \partial_{t} \tilde{F}(t, x, \varepsilon)+\left(\nabla_{x}+\partial_{\varepsilon} \nabla_{x} V\right) . \tilde{J}(t, x, \varepsilon)=0 \\
\tilde{J}(t, x, \varepsilon)=-\tilde{D}(x, \varepsilon)\left(\nabla_{x}+\partial_{\varepsilon} \nabla_{x} V\right) \tilde{F} \\
\tilde{F}(0, x, \varepsilon)=F_{I}(x, \varepsilon) \\
\left.\nabla_{x} V\right) . \tilde{J}(t, x, 0)=0
\end{array}\right.
$$

The diffusion matrix $\tilde{D}(x, \varepsilon)$ is given by $\operatorname{diag}\left(\tilde{D_{11}}, \tilde{D_{22}}\right)$

$$
\tilde{D_{i i}}=2 \int_{\varepsilon_{1}+\varepsilon_{2}=\varepsilon} D_{i i}\left(x, \varepsilon_{1}, \varepsilon_{2}\right) d \varepsilon_{1}=2 \int_{0}^{\varepsilon} D_{i i}\left(x, \varepsilon_{1}, \varepsilon-\varepsilon_{1}\right) d \varepsilon_{1}
$$

and the coefficients $D_{i i}$ are determined in Theorem 2.1. The diffusion matrix $\tilde{D}(x, \varepsilon)$ is strictly positive for $(x, \varepsilon) \in \mathbb{R}^{2} \times \mathbb{R}_{+}^{*}$.

In order to prove Theorem 6.1, we proceed as for Theorem 2.1 and etablish uniform trace estimates. To this aim, the following operator is introduced

$$
\mathcal{B}_{\eta}^{\alpha}=(1-\alpha) \mathcal{B}+\alpha\left[\tilde{\mathcal{B}} \tilde{P}+\frac{1}{1+\eta} \tilde{Q}\right], \quad \eta>0 .
$$

We establish the control of $\gamma_{\alpha}^{\text {out }}(u), \gamma_{\alpha}^{\text {inc }}(u)$ in term of $|u|_{L^{2}\left(\mathcal{O}^{\alpha}\right)}$. We have the following proposition which can be proved by adapting the proof of Proposition 4.1

Proposition 6.1. For all $u \in H^{\alpha}\left(\mathcal{A}, \mathcal{B}_{\eta}^{\alpha}\right)$ and for all $\alpha<2$, we have

i)

$$
\begin{gathered}
\left|\tilde{P} \gamma_{\alpha}^{\text {inc }}(u)\right|_{L^{2}\left(\Gamma^{\alpha}\right)}^{2} \leq\left|\tilde{P} \gamma_{\alpha}^{\text {out }}(u)\right|_{L^{2}\left(\Gamma^{\alpha}\right)}^{2} \leq \frac{2}{(1-K)(2-\alpha(1-K))}(\mathcal{A} u, u)_{L^{2}\left(\mathcal{O}^{\alpha}\right)} \\
\left(1-\frac{1}{1+\eta}\right)\left(2-\alpha\left(1-\frac{1}{1+\eta}\right)\right)\left|\tilde{Q} \gamma_{\alpha}^{\text {out }}(u)\right|_{L^{2}\left(\Gamma^{\alpha}\right)}^{2} \leq 2(\mathcal{A} u, u)_{L^{2}\left(\mathcal{O}^{\alpha}\right)} .
\end{gathered}
$$

ii)

$$
\tilde{Q} \gamma_{\alpha}^{i n c}(u)=\frac{1+\eta(1-\alpha)}{1+\eta} \tilde{Q} \gamma_{\alpha}^{\text {out }}(u)
$$




$$
\left|\tilde{Q} \gamma_{\alpha}^{\text {out }}(u) \sqrt{\Phi_{\alpha, \beta}}\right|_{L_{\delta}^{2}\left(\Gamma^{\alpha}\right)}^{2} \leq C_{1} \alpha|u|_{H^{\alpha}(\mathcal{A})}^{2}+C_{2}\left(1+\frac{\alpha}{\delta}+\frac{1}{\sqrt{\alpha}}\right)|u|_{L^{2}\left(\mathcal{O}^{\alpha}\right)}^{2} .
$$

iii)

$$
\left|P \gamma_{\alpha}^{\text {inc }}(u)\right|_{L^{2}\left(\Gamma^{\alpha}\right)}^{2} \leq\left|P \gamma_{\alpha}^{\text {out }}(u)\right|_{L^{2}\left(\Gamma^{\alpha}\right)}^{2} \leq \frac{2 \alpha}{1-[(1-\alpha) K+\alpha]^{2}}(\mathcal{A} u, u)_{L^{2}\left(\mathcal{O}^{\alpha}\right)} .
$$

iv)

$$
\begin{gathered}
Q \gamma_{\alpha}^{\text {inc }}(u)=(1-\alpha) Q \gamma_{\alpha}^{\text {out }}(u)+\alpha Q \tilde{\mathcal{B}} \tilde{P} \gamma_{\alpha}^{\text {out }}(u)+\frac{\alpha}{1+\eta} \tilde{Q} \gamma_{\alpha}^{\text {out }}(u), \\
\left|Q \gamma_{\alpha}^{\text {out }}(u) \sqrt{\Phi_{\alpha, \beta}}\right|_{L_{\delta}^{2}\left(\Gamma^{\alpha}\right)}^{2} \leq C_{1} \alpha|u|_{H^{\alpha}(\mathcal{A})}^{2}+C_{2}\left(1+\frac{\alpha}{\delta}+\frac{C}{\beta}\right)|u|_{L^{2}\left(\mathcal{O}^{\alpha}\right)}^{2},
\end{gathered}
$$

where $\Phi_{\alpha, \beta}$ is defined in (4.1).

Lemma 6.2. Let $f^{\alpha}$ be the solution of (6.1) constructed as the limit of $f_{\eta}^{\alpha}$ as $\eta \rightarrow 0$. Then $f^{\alpha}$ satisfies the estimates of Lemma 5.1. Moreover, we have:

$$
\begin{gathered}
\left|\tilde{P} \gamma_{\alpha}^{\text {out }}\left(f^{\alpha}\right)(t)\right|_{L^{2}\left(\Gamma^{\alpha}\right)}^{2} \leq C\left|F_{I}\right|_{H^{\alpha}(\mathcal{A})}^{2}, \\
\left|\tilde{P} \gamma_{\alpha}^{\text {inc }}\left(f^{\alpha}\right)(t)\right|_{L^{2}\left(\Gamma^{\alpha}\right)}^{2} \leq C\left|F_{I}\right|_{H^{\alpha}(\mathcal{A})}^{2}, \\
\int_{0}^{T}\left|\tilde{P} \gamma_{\alpha}^{\text {out }}\left(f^{\alpha}\right)(t)\right|_{L^{2}\left(\Gamma^{\alpha}\right)}^{2} d t \leq C \alpha\left|F_{I}\right|_{L^{2}\left(\mathbb{R}^{2} \times \mathbb{R}_{+}\right)}^{2}, \\
\int_{0}^{T}\left|\tilde{P} \gamma_{\alpha}^{\text {inc }}\left(f^{\alpha}\right)(t)\right|_{L^{2}\left(\Gamma^{\alpha}\right)}^{2} d t \leq C \alpha\left|F_{I}\right|_{L^{2}\left(\mathbb{R}^{2} \times \mathbb{R}_{+}\right)}^{2} \\
\int_{0}^{T}\left|\tilde{Q} \gamma_{\alpha}^{\text {out }}\left(f^{\alpha}\right)(t) \sqrt{\Phi_{\alpha, \beta}}\right|_{L_{\delta}^{2}\left(\Gamma^{\alpha}\right)}^{2} d t \leq\left(C_{\delta}+\frac{1}{\beta}\right)\left|F_{I}\right|_{H^{\alpha}(\mathcal{A})},
\end{gathered}
$$

where $C$ denotes generic constants independent of $\alpha, \beta$ and of the data. The function $\Phi_{\alpha, \beta}$ is defined in (4.1).

Proceeding as in Section $5, f^{\alpha}$ can be shown to converge weakly towards a limit $F=F\left(t, x, \varepsilon_{1}, \varepsilon_{2}\right)$. The new estimates (6.14) and (6.15) show that $F$ is actually a function of $\varepsilon=\varepsilon_{1}+\varepsilon_{2}$ ( apply the proof of Lemma 5.2).

Now, we define

$$
\begin{aligned}
\tilde{F}^{\alpha}(t, x, \varepsilon) & =\frac{1}{2 \pi} \int_{0}^{2 \pi} f^{\alpha}(t, x, \sqrt{2 \varepsilon} \cos \theta, \sqrt{2 \varepsilon} \sin \theta) d \theta \\
& =\frac{1}{\pi} \int_{\varepsilon_{1}+\varepsilon_{2}=\varepsilon} F^{\alpha}\left(t, x, \varepsilon_{1}, \varepsilon_{2}\right) \frac{d \varepsilon_{1}}{\sqrt{\varepsilon_{1} \varepsilon_{2}}}
\end{aligned}
$$




$$
\begin{aligned}
\tilde{J}_{1}^{\alpha}(t, x, \varepsilon) & =\frac{1}{\alpha} \int_{0}^{2 \pi} f^{\alpha}(t, x, \sqrt{2 \varepsilon} \cos \theta, \sqrt{2 \varepsilon} \sin \theta) \sqrt{2 \varepsilon} \cos \theta d \theta \\
& =\int_{\varepsilon_{1}+\varepsilon_{2}=\varepsilon} J_{1}^{\alpha}\left(t, x, \varepsilon_{1}, \varepsilon_{2}\right) d \varepsilon_{1}, \\
\tilde{J}_{2}^{\alpha}(t, x, \varepsilon) & =\frac{1}{\alpha} \int_{0}^{2 \pi} f^{\alpha}(t, x, \sqrt{2 \varepsilon} \cos \theta, \sqrt{2 \varepsilon} \sin \theta) \sqrt{2 \varepsilon} \sin \theta d \theta \\
& =\int_{\varepsilon_{1}+\varepsilon_{2}=\varepsilon} J_{2}^{\alpha}\left(t, x, \varepsilon_{1}, \varepsilon_{2}\right) d \varepsilon_{1},
\end{aligned}
$$

where $F^{\alpha}, J_{1}^{\alpha}$ and $J_{2}^{\alpha}$ are defined by (5.11), (5.12) and (5.13). We have

Lemma 6.3. Let $f^{\alpha}$ be the solution of (6.1). For any test function $\phi \in C_{c}^{1}([0, T] \times$ $\left.\mathbb{R}^{2} \times \mathbb{R}_{+}\right)$such that $\phi(T, \cdot, \cdot)=0$, we have

$$
\begin{aligned}
& \int_{0}^{T} \int_{\Omega^{\alpha} \times \mathbb{R}_{+}}\left[2 \pi \tilde{F}^{\alpha}(t, x, \varepsilon) \alpha \partial_{t} \phi(t, x, \varepsilon)+\alpha \tilde{J}_{1}^{\alpha}(t, x, \varepsilon)\left(\partial_{x_{1}}+\partial_{x_{1}} V \partial_{\varepsilon}\right) \phi(t, x, \varepsilon)\right] d x d \varepsilon d t \\
& \left.\quad+\int_{0}^{T} \int_{\Omega^{\alpha} \times \mathbb{R}_{+}} \alpha \tilde{J}_{2}^{\alpha}(t, x, \varepsilon)\left(\partial_{x_{2}}+\partial_{x_{2}} V \partial_{\varepsilon}\right) \phi(t, x, \varepsilon)\right) d x d \varepsilon d t \\
& \quad+\alpha \int_{\mathbb{R}^{2} \times \mathbb{R}_{+}} 2 \pi F_{I}(x, \varepsilon) \phi(0, x, \varepsilon) d x d \varepsilon=0
\end{aligned}
$$

Since $\lim \tilde{F}^{\alpha}=\lim f^{\alpha}$, and $\lim \tilde{J}_{i}^{\alpha}=\tilde{D}_{i i}(x, \varepsilon)\left(\nabla_{x_{i}}+\partial_{\varepsilon} \nabla_{x_{i}} V\right) \tilde{F}$, we can pass to the limit in (6.20) and find the weak formulation of (6.3). Namely,

Corollary 6.1. For any test function $\phi \in C_{c}^{2}\left([0, T] \times \mathbb{R}^{2} \times \mathbb{R}_{+}\right)$such that $\phi(T, \cdot, \cdot)=$ 0 , we have

$$
\begin{aligned}
& \int_{0}^{T} \int_{\mathbb{R}^{2} \times \mathbb{R}_{+}}\left[2 \pi \tilde{F}(t, x, \varepsilon) \partial_{t} \phi(t, x, \varepsilon)+\tilde{J}_{1}(t, x, \varepsilon)\left(\partial_{x_{1}}+\partial_{x_{1}} V \partial_{\varepsilon}\right) \phi(t, x, \varepsilon)\right] d x d \varepsilon d t \\
& \left.+\int_{0}^{T} \int_{\mathbb{R}^{2} \times \mathbb{R}_{+}} \tilde{J}_{2}(t, x, \varepsilon)\left(\partial_{x_{2}}+\partial_{x_{2}} V \partial_{\varepsilon}\right) \phi(t, x, \varepsilon)\right) d x d \varepsilon d t \\
& +\int_{\mathbb{R}^{2} \times \mathbb{R}_{+}} 2 \pi F_{I}(x, \varepsilon) \phi(0, x, \varepsilon) d x d \varepsilon=0 .
\end{aligned}
$$

Acknowledgments. The LAMSIN's researchers work is supported by the Tunisian Secretary of State for Research and Technology (SERST) within the LAB-STI-02 program. This research has been partially supported by the cooperation project entitled "Modélisation et analyse mathématique de problèmes de transport dans les semiconducteurs" \#99/R 1503 run by the CNRS and the DGRST.

\section{REFERENCES}

[1] C. Bardos, Problèmes aux limites pour les équations aux dérivées partielles du premier ordre à coefficients réels; Théorèmes d'approximation; application à l'équation de transport, Ann. Scient. Ec. Nom. Sup., 4:185-233, 1970.

[2] N. Ben Abdallah and P. Degond, On a hierarchy of macroscopic models for semiconductors, J. Maths. Phys., 37:3306-3333, 1996.

[3] N. Ben Abdallah, P. Degond, and P.A. Markowich, On a one-dimensional Schrödinger-Poisson Scattering model, ZAMP, 48:135-155, 1997. 
[4] N. Ben Abdallah, P. Degond, A. Mellet, and F. Poupaud, Electron transport in semiconductor superlattices, Quarterly Appl. Math., 61(1):161-192, 2003.

[5] V. Bryanzari, G. Korotchenkov, and S. Dmitriev, Simulation of thin film gas sensors kinetics, Sens. Actuators, B, 61:143-153, 1999.

[6] P. Degond, A model of near-wall conductivity and its application to plasma thrusters, SIAM J. Appl. Math., 58:1138-1162, 1998.

[7] P. Degond, Transport of trapped particles in a surface potential, Elsevier. Stud. Math. Appl., 31:273-296, 2002.

[8] P. Degond, An infinite system of diffusion equations arising in transport theory: The coupled spherical Harmonic Expansion Model, Math. Mod. Meth. Appli. Sci., 11(5):903-932, 2001.

[9] P. Degond, V. Latocha, S. Mancini, and A. Mellet, Diffusion dynamics of an electron gas confined between two plates, Methods Appl. Anal., 9(1):127-150, 2002.

[10] P. Degond, D. Levermore, and C. Schmeiser, A note on the Energy-Transport limit of the semiconductor Boltzmann equation, to appear in the IMA volumes in Mathematics and its applications, 2004.

[11] P. Degond, A. Nouri, and C. Schmeiser, Macroscopic models for ionization in the presence of strong electric fields, Transp. Theory and Stat. Phys., 551-561, 2000.

[12] P. Degond and S. Mancini, Diffusion driven by collisions with the boundary, Asymptotic Analysis, 27(1):47-73, 2001.

[13] P. Degond, V. Latocha, L. Garrigues, and J.P. Boeuf, Electron Transport in atationary plasma Trusters, Transp. Theory and Stat. Phys., 27:203-221, 1998.

[14] P. Degond and K. Zhang, Diffusion approximation of a scattering matrix model of semiconductor superlattices, SIAM J. Appl. Math., 63(1):279-298, 2002.

[15] P. Degond and K. Zhang, A scattering matrix model of semiconductor superlattices in multidimensional wave vector space and the diffusion limit, Chin. Ann. Math., 24(B):167-190, 2003.

[16] P. Dmitruk, A. Saul, and L. Reyna, High electric field approximation in semiconductor devices, Appl. Math. Letters, 5:99-102, 1992.

[17] A. Gnudi, D. Ventura, and G. Baccarani, Modelling impact ionization in a BJT by means of spherical harmonics expansion of the Boltzmann Transport Equation, IEEE Trans CAD of integ., circuits and systems, 12:1706-1713, 1993.

[18] A. Gnudi, D. Ventura, G. Baccarani, and F. Odeh Two-dimensional MOSFET simulation by means of a multidimensional harmonic expansion of the Boltzmann transport equation, Solid State Electron, 36:575-581, 1993.

[19] N. Goldsman, L. Henrickson, and J. Frey, A physics based analytical numerical solution to the Boltzmann transport equation for use in device simulation, Solid State Electron, 34:389396, 1991.

[20] F. Greuter and G. Blatter, Electrical properties of grains boundaries in polycrystalline compound semiconductors, Review article, Semicond. Sci. Technl., 5:111-137, 1990.

[21] Y. Mishin and Chr. Herzig, Grain boundary diffusion: recent progress ans future resaerch, Materials Science and Ingineering, A, 260:55-71, 1999.

[22] G. Nguetseng, A general convergence result for a functional related to the theory of homogenization, SIAM Math. Anal., 20:608-623, 1989.

[23] A. Pazy, Semigroups of Linear Operators and Applications to Partial Differential Equations, Springer, New York, 1983.

[24] G.E. Pike and C.H. Seager, The de voltage dependence of semiconductor grain-boundary resistance, J. Appl. Phys., 5(50):3414-3422, 1979.

[25] C. Schmeiser, and A. Zwirchmayr, Elastic and drift-diffusion limits of electron-phonon interaction in semiconductors, Math. Mod. Methj. Appli. Sci., 8:37-54, 1998.

[26] D. Ventura, A. Gnudi, G. Baccarani, and F. Odeh, Multidimensional spherical harmonics expansion of Boltzmann equation for transport in semiconductors, Appl. Math. Letters, 5:85-90, 1992.

[27] T.H. Wolkenstein, Physico-Chimie de la Surface des Semi-Conducteurs, Edition Mir, 1973. 